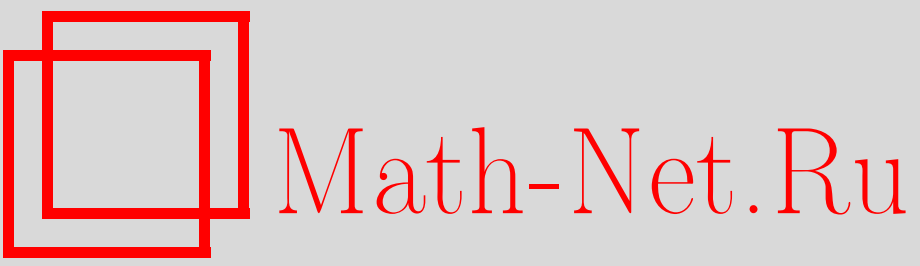

А. В. Маршаков, Об интегрируемых системах и суперсимметричных калибровочных теориях, ТМФ, 1997, том 112, номер 1, 3-46

DOI: https://doi.org/10.4213/tmf1025

Использование Общероссийского математического портала Math-Net.Ru подразумевает, что вы прочитали и согласны с пользовательским соглашением

http://www.mathnet.ru/rus/agreement

Параметры загрузки:

IP : 35.173 .219 .149

26 апреля 2023 г., 10:55:24 
ТЕОРЕТИЧЕСКАЯ

И МАТЕМАТИЧЕСКАЯ

ФИЗИКА

Том 112, № 1

июль, 1997

А. В. Маршаков*

\title{
ОБ ИНТЕГРИРУЕМЫХ СИСТЕМАХ И СУПЕРСИММЕТРИЧНЫХ КАЛИБРОВОЧНЫХ ТЕОРИЯХ ${ }^{1)}$
}

\begin{abstract}
Обсуждаются свойства $\mathcal{N}=2$ суперсимметричных калибровочных теорий, лежащие в основе гипотез Виттена-Зайберга. Рассматриваются основные моменты теории конечнозонных решений интегрируемых уравнений в терминах римановых поверхностей и производящих дифференциалов, поясняется инвариантный смысл этих определений. Этот формализм применяется к формулировке недавно найденных непертурбативных решений $\mathcal{N}=2$ суперсимметричных неабелевых калибровочных теорий. В известных случаях производится сравнение с результатами, которые могут быть получены стандартными методами квантовой теории поля.
\end{abstract}

\section{1. ВВЕДЕНИЕ. ОСНОВНЫЕ ОПРЕДЕЛЕНИЯ}

Основной целью данной работы является доступное, насколько это возможно, пояснение связи между точными непертурбативными решениями $\mathcal{N}=2$ суперсимметричной неабелевой калибровочной теории (возникающей в полевом пределе теории струн) и интегрируемыми системами. Эта связь была впервые замечена в [1] и исследовалась с разных точек зрения в работах [2-19], где при желании можно найти все интересные детали.

План данной статьи выглядит следуюшим образом. Сначала обсуждается то, что известно об эффективных низкоэнергетических действиях $\mathcal{N}=2$ суперсимметричных калибровочных теорий, а также приводятся основные понятия и определения алгебро-геометрических решений интегрируемых систем в терминах комплексных кривых и производяшего 1-дифференциала. Эти понятия детально обсуждаются на основном примере - периодической задаче для цепочки Тоды. Далее мы переходим непосредственно к эффективным теориям Виттена-Зайберга, показывая, что они действительно определяются теми же самыми данными, что и конечнозонные интегрируемые системы, хотя их полная формулировка требует рассмотрения деформаций конечнозонных решений интегрируемых уравнений. Наконец, рассматриваются явные дифференциальные

\footnotetext{
1) Статья написана по заказу Редколлегии.
}

\footnotetext{
* Отделение теоретической физики Физического института им. П.Н. Лебедева, Институт теоретической и экспериментальной физики, Москва, Россия. E-mail: mars@lpi.ac.ru, andrei@heron.itep.ru, marshakov@nbivms.nbi.dk
} 
уравнения, которым удовлетворяют препотенциалы эффективных теорий, и явные вычисления препотенциалов исходя из этих уравнений сравниваются с хорошо известными вычислениями в суперсимметричных калибровочных теориях.

1.1. Эффективная теория Виттена-Зайберга. Начнем с некоторых предварительных сведений, которые будут использованы ниже для формулировки эффективной теории Виттена-Зайберга в терминах интегрируемых систем.

Основным объектом изучения являются эффективные (абелевы) $\mathcal{N}=2$ суперсимметричные калибровочные теории в четырех- (или пяти-) мерии ${ }^{2}$, отвечаюшие безмассовым возбуждениям в $\mathcal{N}=2$ суперсимметричной неабелевой калибровочной теории с затравочным действием

$$
\mathcal{L}=\int d^{4} \theta \widehat{\mathcal{L}}(\mathbf{\Phi})=\cdots \frac{1}{g^{2}} \operatorname{Tr} \mathbf{F}_{\mu \nu}^{2}+i \theta \operatorname{Tr} \mathbf{F}_{\mu \nu} \widetilde{\mathbf{F}}_{\mu \nu}+\cdots
$$

Точные непертурбативные результаты [20-23] содержат информацию о массивных БПЗ-состояниях ${ }^{3)}$ (БПЗ - Богомольный-Прасад-Зоммерфельд) (" $W$-бозонах" и монополях) и вильсоновском эффективном действии для безмассовых частиц (см., например, [24]). Наиболее сушественной особенностью этой эффективной теории является то, что эффективное действие может быть записано с помошью одной (голоморфной) функции нескольких комплексных переменных [20, 21]. В дальнейшем, следуя принятой терминологии, эту функцию будем называть препотенциалом эффективной теории.

Для $\mathcal{N}=2$ суперсимметричной калибровочной теории скалярный потенщиал имеет вид $V(\phi)=\operatorname{Tr}\left[\phi, \phi^{\dagger}\right]^{2}$ и его минимумы с точностью до калибровочных преобразований отвечают в теории с группой $S U\left(N_{c}\right)$ диагональным бесследовым матрицам

$$
\phi=\left(\begin{array}{llll}
A_{1} & & & \\
& A_{2} & & \\
& & \ddots & \\
& & & A_{N_{c}}
\end{array}\right)
$$

инварианты которых

$$
\operatorname{det}(\lambda-\phi)=P_{N_{c}}(\lambda)=\sum_{k=0}^{N_{c}} s_{N_{c}-k} \lambda^{k}
$$

(или любой другой набор алгебраически независимых инвариантов) в количестве, равном рангу группы $\left(\operatorname{rank} S U\left(N_{c}\right)=N_{c}-1\right)$, параметризуют пространство параметров теории, которые принято называть физическими модулями. Эффект Хиггса приводит к появлению массы у внедиагональной части калибровочного поля $\mathbf{A}_{\mu}$ :

$$
\left[\boldsymbol{\phi}, \mathbf{A}_{\mu}\right]_{i j}=\left(A_{i}-A_{j}\right) \mathbf{A}_{\mu}^{i j}
$$

\footnotetext{
${ }^{2)}$ Отметим сразу, что в рассматриваемую эффективную формулировку точных непертурбативных решений размерность пространства-времени входит весьма неявным образом. Предлагаемая эффективная формулировка в терминах интегрируемых систем универсальна и известна на сегодняшний день для $2 d$-струнных моделей, а также теорий, рассматриваемых в основном тексте статьи.

3) БПЗ-состояниями называются состояния, относящиеся к "минимальным" мультиплетам, массы которых пропорциональны центральным зарядам расширенной $\mathcal{N} \geq 2$ алгебры суперсимметрии.
} 
тогда как диагональная часть остается безмассовой, т.е. калибровочная группа $G=S U\left(N_{c}\right)$ нарушается до $U(1)^{\operatorname{rank} G}=U(1)^{N_{c}-1}$. Таким образом безмассовый сектор может быть представлен как $\mathcal{N}=2$ абелева калибровочная теория, эффективный лагранжиан которой определяется в терминах суперполей

$$
\Phi_{i}=\varphi^{i}+\vartheta \sigma_{\mu \nu} \widetilde{\vartheta} f_{\mu \nu}^{i}+\cdots, \quad \sigma_{\mu \nu} \sim\left[\gamma_{\mu}, \gamma_{\nu}\right]
$$

вакуумные значения последних совпадают с диагональными элементами матрицы (2). Поэтому функция комплексных переменных

$$
\mathcal{F}(a)=\left.\mathcal{F}(A)\right|_{\sum A_{i}=0}
$$

действительно определяет вильсоново эффективное действие безмассовых полей, которое получается из нее подстановкой

$$
\mathcal{L}_{\text {eff }} \sim \operatorname{Im} \int d^{4} \vartheta \mathcal{F}\left(A_{i} \rightarrow \Phi_{i}\right)=\ldots \operatorname{Im} \frac{\partial^{2} \mathcal{F}}{\partial a_{i} \partial a_{j}} f_{\mu \nu}^{i} f_{\mu \nu}^{j}+\cdots
$$

Этот факт можно проверить явным вычислением квантовых поправок в $\mathcal{N}=2$ суперсимметричной калибровочной теории.

Что касается массивных возбуждений в $\mathcal{N}=2$ неабелевой калибровочной теории, то оказывается [20, 21], что, по крайней мере, спектр БПЗ-состояний связан с функцией $\mathcal{F}$ соотношением $M \sim\left|\mathbf{n a}+\mathbf{m a}_{D}\right|$, где $\mathbf{a}_{D}=\partial \mathcal{F} / \partial \mathbf{a}$. Согласно гипотезе Виттена-Зайберга (которая будет строго сформулирована ниже, в п. 1.3) массы БПЗ-состояний $\mathbf{a}$ и $\mathbf{a}_{D}$ являются периодами мероморфного дифференциала на вспомогательной римановой поверхности и зависят от вакуумных средних теории, которые параметризуют пространство модулей вспомогательной поверхности. Например, для чистой калибровочной теории с группой $S U\left(N_{c}\right)$ кривая и соответствуюший мероморфный дифференциал имеют вид [20, 22]

$$
w+\frac{1}{w}=2 P_{N_{c}}(\lambda), \quad d S=\lambda \frac{d w}{w},
$$

а для $\mathcal{N}=2$ суперсимметричной хромодинамики $[21,23]$

$$
W+\frac{1}{W}=\frac{2 P_{N_{c}}(\lambda)}{P_{N_{f}}(\lambda)}, \quad d S=\lambda \frac{d W}{W} .
$$

Таким образом, именно знание функции $\mathcal{F}$ комплексных переменных как функции модулей и ее всевозможных производных, например разложение по некоторым внешним источникам $\mathbf{T}$, дает наиболее полную непертурбативную информацию о теории. Ниже будет продемонстрировано, что главной целью являются нахождение и исследование свойств производящей функшии $\mathcal{F}$, которая по части своих переменных имеет зависимость от модулей комплексной структуры вспомогательной римановой поверхности $(7),(8)$. 
1.2. Интегрируемые системы. Основной идеей, как уже было сказано, является отождествление функции $\mathcal{F}$, а также других характеристик физической теории с величинами, имеющими смысл в системах интегрируемых уравнений типа уравнений Кадомцева-Петвиашвили (КП) и Тоды (КП/Тоды). Для того чтобы сформулировать эту связь, приведем также некоторые определения из теории интегрируемых моделей. Уравнения, возникающие в классе рассматриваемых задач, относятся к иерархиям уравнения КП

$$
\frac{\partial^{2} U}{\partial T_{2}^{2}}=\frac{\partial}{\partial T_{1}}\left(\frac{\partial U}{\partial T_{3}}+U \frac{\partial U}{\partial T_{1}}+\frac{\partial^{3} U}{\partial T_{1}^{3}}\right)
$$

и двумерной решетки Тоды

$$
\frac{\partial^{2} \phi_{n}}{\partial T_{1} \partial \bar{T}_{1}}=e^{\phi_{n+1}-\phi_{n}}-e^{\phi_{n}-\phi_{n-1}}
$$

(а также к другим аналогичным иерархиям, которые будут называться в дальнейшем иерархиями уравнений типа уравнений КП/Тоды), а точнее к их редукциям. Понятие иерархии означает, что динамические системы (9) и (10) обладают бесконечным количеством интегралов движения, которым можно сопоставить бесконечное количество взаимно коммутирующих (и коммутирующих с первыми уравнениями, определяемыми формулами (9) и (10)) потоков. Дифференциальные уравнения по старшим временам $T_{k}$ имеют более сложный вид, если их писать как уравнения на функции $U(\mathbf{T})$ и $\phi_{n}(\mathbf{T})$, однако существует более изящный способ задания всей иерархии.

Этот способ основан на вспомогательной линейной задаче для иерархии интегрируемых уравнений

$$
\frac{\partial}{\partial T_{k}} \Psi=B_{k} \Psi
$$

где $B_{k}=B_{k}[U ; \phi]$ - дифференциальные операторы только по $T_{1}$ в случае уравнения КП (9) или разностные операторы по дискретному времени $n$ в случае уравнения Тоды (10), а решение $\Psi$ вспомогательной линейной задачи называется обычно функцией Бейкера-Ахиезера. К уравнениям (11) можно добавить уравнение Лакса

$$
\mathcal{L} \Psi=\lambda \Psi
$$

которое при редукциях возникает как одно из уравнений цепочки (11). При этом иерархия нелинейных интегрируемых уравнений эквивалентна уравнениям Лакса

$$
\frac{\partial \mathcal{L}}{\partial T_{k}}=\left[B_{k}, \mathcal{L}\right]
$$

или условиям совместности (Захарова-Шабата)

$$
\left[\frac{\partial}{\partial T_{k}}-B_{k}, \frac{\partial}{\partial T_{l}}-B_{l}\right]=0 .
$$

Наиболее универсальным объектом в такой формулировке интегрируемых задач является $\tau$-функция Хироты, удовлетворяюшая бесконечной цепочке билинейных дифференциальных (разностных) уравнений и генерируюшая решения интегрируемой иерархии, функция Бейкера-Ахиезера и т.п. Например, для иерархии КП функция Бейкера-Ахиезера и решения иерархии выражаются через $\tau$-функцию по формулам (ниже 
в тексте $\tau$-функция будет обозначаться буквой $\mathcal{T}$, чтобы не путать ее со стандартным обозначением для модулярного параметра тора)

$$
\begin{gathered}
\Psi=e^{\sum T_{k} \lambda^{k} \frac{\mathcal{T}\left(T_{k}-\frac{1}{k \lambda^{k}}\right)}{\mathcal{T}(\mathbf{T})}} \\
U(\mathbf{T})=\partial^{2} \log \mathcal{T}(\mathbf{T}) \equiv \frac{\partial^{2}}{\partial T_{1}^{2}} \log \mathcal{T}(\mathbf{T}) \ldots
\end{gathered}
$$

Аналогичные формулы существуют и для других иерархий.

Иерархии Тоды и КП имеют бесконечное число решений, параметризуемых так называемой точкой бесконечномерного грассманиана [25-27] или, грубо говоря, функцией двух переменных. Частные решения можно выделять дополнительными условиями, часто имеющими вид дополнительных (как правило, линейных) уравнений на $\tau$-функцию.

Особую роль играют конечномерные редукции иерархий интегрируемых уравнений, когда только конечное число интегралов движения и потоков $\partial / \partial T_{k}$ является независимым. Красивый пример конечномерных редукций иерархий уравнений КП/Тоды - так называемые конечнозонные решения, определяемые условиями

$$
\begin{gathered}
{[\mathcal{L}, \mathcal{A}]=0} \\
\mathcal{A}=\sum_{k}^{\text {finite }} c_{k} B_{k}
\end{gathered}
$$

где $\mathcal{L}$ - оператор Лакса (12), $B_{k}$ - операторы эволюции функции Бейкера-Ахиезера (11), а $c_{k}$ - некоторый конечны й набор ненулевых констант. Интегрирование конечнозонных решений называется конструкцией Кричевера [28] и сводится к следуюшим шагам ${ }^{4)}$ :

1) совместный спектр коммутирующих операторов $\mathcal{L}$ и $\mathcal{A}(16)$ задается системой уравнений, описывающих комплексную кривую $\Sigma$, в простейшем случае $\mathcal{P}(\mathcal{L}, \mathcal{A})=0$;

2 ) функция Бейкера-Ахиезера является сечением некоторого расслоения над $\Sigma$ в используемых ниже случаях почти всегда линейного расслоения;

3) модули комплексной кривой суть интегралы движения системы (16);

4) интегрируюшей заменой переменных является преобразование Абеля, тор Лиувилля (угловые переменные) - вешественное сечение якобиана кривой $\Sigma$;

5) гамильтонова структура конечнозонного решения формулируется с помощњю производяшего мероморфного 1-дифференшиала $d S$, периоды которого (интегралы по нетривиальным циклам на римановой поверхности) являются переменными действия (каноническим набором интегралов движения) системы.

Возникающие при этом комплексные кривые задаются алгебраическими уравнениями вида

$$
\mathcal{P}(\lambda, w)=0
$$

\footnotetext{
4) Мы приводим здесь лишь "грубую" картину конечнозонного интегрирования исключительно для пояснения утверждений, сделанных в основном тексте, отсылая за точными математическими формулировками к [27-31].
} 
(одно соотношение вида (17) на две переменные, где полином $\mathcal{P}$, коэффициенты которого представляют собой модули комплексной структуры, задает одномерное комплексное (или двумерное вешественное) многообразие) или системами уравнений на несколько комплексных переменных. Топологически каждая комплексная кривая характеризуется единственным параметром - родом $g$ (количеством приклеенных "ручек"). При этом для поверхности фиксированного рода $\Sigma_{g}$ комплексная структура определяется $3 g-3$ параметрами - модулями комплексной структуры, т.е. $\operatorname{dim}_{\mathbf{C}} \mathcal{M}_{g}=3 g-3$. Конечнозонным интегрируемым системам обычно отвечают $g$-параметрические семейства комплексных кривых (так чтобы размерность пространства модулей, равная количеству независимых интегралов движения, совпадала с размерностью якобиана кривой, т.е. числом угловых переменных $)^{5)}$. Размерность же якобиана совпадает с количеством глобально-определенных голоморфных дифференциалов $d \omega_{i}, i=1, \ldots, g$, и равна роду поверхности. На поверхности рода $g$ сушествуют $2 g$ независимых нестягиваемых циклов (по два вокруг каждой "ручки”), канонический набор которых отвечает разбиению на так называемые $A_{i}, i=1, \ldots, g$, и $B_{i}, i=1, \ldots, g$, циклы с индексом пересечения $A_{i} \circ B_{j}=\delta_{i j}$. Голоморфные дифференциалы канонически выбираются нормированными на А-циклы:

$$
\oint_{A_{j}} d \omega_{i}=\delta_{i j}
$$

При этом интегралы по В-циклам дают матрицу периодов

$$
\begin{gathered}
\oint_{B_{j}} d \omega_{i}=T_{i j}, \\
\int_{\Sigma_{g}} d \omega_{i} \wedge \overline{d \omega}_{j}=\operatorname{Im} T_{i j} .
\end{gathered}
$$

Как хорошо известно, матрица периодов (19) является симметричной, что легко проверяется применением теоремы Стокса

$$
0=\int_{\Sigma_{g}} d \omega_{i} \wedge d \omega_{j}=\sum_{k=1}^{g} \oint_{A_{k}} d \omega_{i} \oint_{B_{k}} d \omega_{j}-(i \leftrightarrow j)=T_{i j}-T_{j i} .
$$

Производные производяшего дифференциала $d S$ по $g$-направлениям в пространстве модулей, отвечаюшим интегралам движения, являются (не обязательно каноническими) голоморфными дифференциалами

$$
\frac{\partial d S}{\partial h_{k}} \sim d v_{k}
$$

\footnotetext{
5) Строго говоря, $g$-параметрические семейства возникают в простейших случаях (в данном контексте, например, для калибровочных теорий с группами $S U\left(N_{c}\right)$, при этом $\left.g=\operatorname{rank} G\right)$. В более общей ситуации следует рассматривать многообразия Прима, возникающие в результате "факторизации" якобиана по некоторой инволюции. Вообше говоря, для произвольной группы $g \geq \operatorname{rank} G$, но при этом только $\operatorname{rank} G$ контуров (и соответственно $\operatorname{rank} G$ параметров) определяет соответствуюшую интегрируемую систему (см., например, [17]).
} 
При этом канонические (нормированные) голоморфные дифференциалы появляются в правой части (21), если в качестве соответствующих координат на пространстве модулей выбрать канонически нормированные переменные действия - $\mathbf{A}$-периоды дифференциала $d S$ :

$$
\mathbf{a}=\oint_{\mathbf{A}} d S .
$$

Соответствующие им “дуальные” $\mathbf{B}$-периоды принято обозначать $\mathbf{a}_{D}$ :

$$
\mathbf{a}_{D}=\oint_{\mathbf{B}} d S .
$$

Сушествование соотношения (21) тривиально проверяется для известных примеров семейств комплексных кривых, вообе говоря, оно связано с существованием специальных направлений в пространстве модулей таких, что удовлетворяется условие

$$
\frac{\partial d v_{k}}{\partial h_{l}}=\frac{\partial d v_{l}}{\partial h_{k}} .
$$

K этому свойству мы вернемся ниже в контексте иерархий уравнений Уизема.

Из имеющих отношение к непертурбативным квантовым теориям конечнозонные решения являются наиболее простыми решениями интегрируемых систем. Вообше говоря, они представляют собой лишь некоторое приближение к точным решениям квантовой теории, позволяя при этом получить часть информации о физических характеристиках эффективной теории, в основном касающихся ее безмассового спектра. Кроме того, во многих случаях (например в $2 d$-теории струн) точные решения можно рассматривать как интегрируемые деформации конечнозонных решений, описываемые иерархиями уравнений Уизема.

1.3. Отображение Виттена-Зайберга. Теперь можно перейти к точной формулировке эффективной теории Виттена-Зайберга, формально определяемой как отображение

$$
G, \tau, h_{k} \rightarrow a_{i}, a_{i}^{D}
$$

где $G$ - калибровочная группа, $\tau$ - ультрафиолетовая константа связи, а $h_{k}=\frac{1}{k}\left\langle\operatorname{Tr} \Phi^{k}\right\rangle-$ вакуумные значения хиггсовских полей, и изяшно описываемой с помошью интегрируемой системы. В большинстве случаев интегрируемая система строится по спектральной кривой $\Sigma_{g}$ рода $g \geq \operatorname{rank} G$, так что параметры $h_{k}$ являются некоторыми специальными (в большинстве случаев "гиперэллиптическими") модулями комплексной структуры. Отображение (25) задается $2 g$ периодами (22), (23) мероморфной 1-формы (21), которые определяют БПЗ-массивньй спектр

$$
M \sim\left|\mathbf{n a}+\mathbf{m a}^{D}\right|
$$

и препотенциал $\mathcal{F}$

$$
a_{i}^{D}=\frac{\partial \mathcal{F}}{\partial a_{i}} .
$$


При этом вторые производные препотенциала являются матричными элементами матрицы периодов комплексной кривой, а с физической точки зрения они отвечают низкоэнергетическим константам связи в эффективной абелевой теории (ср. с (6)): действительно, из (22), (23) и (19) следует, что

$$
\begin{gathered}
\delta_{i j}=\frac{\partial a_{i}}{\partial a_{j}}=\oint_{A_{i}} \frac{\partial d S}{\partial a_{j}}=\oint_{A_{i}} d \omega_{j}, \\
\frac{\partial^{2} \mathcal{F}}{\partial a_{i} \partial a_{j}}=\frac{\partial a_{i}^{D}}{\partial a_{j}}=\oint_{B_{i}} \frac{\partial d S}{\partial a_{j}}=\oint_{B_{i}} d \omega_{j}=T_{i j} .
\end{gathered}
$$

Римановы поверхности $\Sigma_{g}$ оказываются специальными комплексными кривыми - спектральными кривыми конечнозонных интегрируемых систем, а именно периодической цепочки Тоды и ее естественных обобщений, а основной объект в теории препотенциал

$$
\mathcal{F}=\log \mathcal{T}
$$

является логарифмом $\tau$-функции иерархии уравнений Уизема, ассоциированной с соответствуюшим конечнозонным решением.

\section{2. ЦЕПОЧКА ТОДЫ: ПЕРИОДИЧЕСКАЯ ЗАДАЧА}

Продемонстрируем теперь приведенные выше формулы для простейшего случая периодической цепочки Тоды, которая с точки зрения точных непертурбативных решений отвечает $4 d$ - чистой $\mathcal{N}=2$ суперсимметричной неабелевой калибровочной теории (или $\mathcal{N}=2$ суперсимметричной глюодинамике) $[22,1]$. Периодическая задача в цепочке Тоды может быть сформулирована двумя различными эквивалентными способами, каждый из которых естественным образом “деформируется", причем лишь одним из двух возможных способов. Эти деформации гипотетически отвечают включению взаимодействия $4 d$-калибровочной теории с $\mathcal{N}=2$ гипермультиплетами материи в присоединенном и фундаментальном представлениях калибровочной группы, соответственно $[4-8,11,12]$.

Цепочкой Тоды называется простейшая система частиц, в которой только соседние частицы взаимодействуют друг с другом по экспоненциальному закону и которая определяется уравнениями движения

$$
\frac{\partial \phi_{i}}{\partial t}=p_{i}, \quad \frac{\partial p_{i}}{\partial t}=e^{\phi_{i+1}-\phi_{i}}-e^{\phi_{i}-\phi_{i-1}}
$$

где следует предположить (для периодической задачи с "периодом" $N_{c}$ ), что $\phi_{i+N_{c}}=\phi_{i}$ и $p_{i+N_{c}}=p_{i}$. Цепочка Тоды является вполне интегрируемой системой с $N_{c}$ "гамильтонианами" (переменными действия), коммутирующими относительно скобки Пуассона, $h_{1}^{T C}=\sum p_{i}, h_{2}^{T C}=\sum\left(\frac{1}{2} p_{i}^{2}+e^{\phi_{i}-\phi_{i-1}}\right)$ и т.д. Как и для любой конечнозонной системы, периодическая задача для цепочки Тоды может быть сформулирована в терминах (собственных значений и собственных функций) двух операторов: оператора Лакса (12) $\mathcal{L}$ (или вспомогательной линейной задачи для $(30)$ )

$$
\lambda \psi_{n}^{ \pm}=\sum_{k} \mathcal{L}_{n k} \psi_{k}^{ \pm}=e^{\frac{1}{2}\left(\phi_{n+1}-\phi_{n}\right)} \psi_{n+1}^{ \pm}+p_{n} \psi_{n}^{ \pm}+e^{\frac{1}{2}\left(\phi_{n}-\phi_{n-1}\right)} \psi_{n-1}^{ \pm}\left(= \pm \frac{\partial}{\partial t} \psi_{n}^{ \pm}\right)
$$


и $\mathcal{A}$-оператора $(16)$, который в данном случае удобно выбрать как оператор монодромии - сдвига по дискретной переменной "номера" частицы

$$
T \phi_{n}=\phi_{n+N_{c}}, \quad T p_{n}=p_{n+N_{c}}, \quad T \psi_{n}=\psi_{n+N_{c}} .
$$

Условие совместного спектра этих двух операторов ${ }^{6)}$

$$
\mathcal{L} \psi=\lambda \psi, \quad T \psi=w \psi, \quad[\mathcal{L}, T]=0
$$

означает, что между ними сушествует соотношение $\mathcal{P}(\mathcal{L}, T)=0$, которое строго формулируется в качестве соотношения на спектр - спектральную кривую $\Sigma: \mathcal{P}(\lambda, w)=0$. Производящая функция для интегралов движения может быть выражена через операторы $\mathcal{L}$ и $T$, и для цепочки Тоды сушествуют два различных представления такого типа.

В первом варианте оператор Лакса (31) записывается в базисе собственных функций $T$-оператора. Для цепочки длины $N_{c}$ он становится при этом матрицей размера $N_{c} \times N_{c}$ :

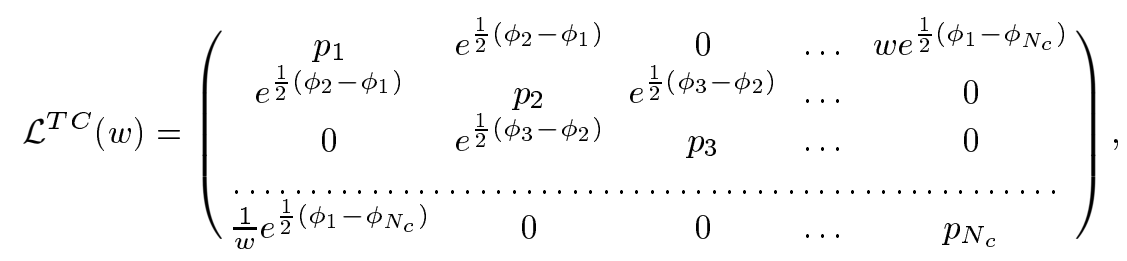

определенной на цилиндре. Матрица (34), как следует из (31), является трехдиагональной, за исключением "угловых" матричных элементов, возникающих благодаря периодическим граничным условиям (32), редуцируюшим матрицу, вообще говоря, бесконечного размера (31) до конечной, но зависящей от спектрального параметра $w$. Собственные числа оператора Лакса, определяемые спектральным уравнением

$$
\mathcal{P}(\lambda, w)=\operatorname{det}_{N_{c} \times N_{c}}\left(\mathcal{L}^{T C}(w)-\lambda\right)=0,
$$

коммутируют друг с другом относительно скобки Пуассона. Подставляя явное выражение (34) в (35), получаем [32]

$$
w+\frac{1}{w}=2 P_{N_{c}}(\lambda)
$$

или

$$
y^{2}=P_{N_{c}}^{2}(\lambda)-1, \quad 2 y=w-\frac{1}{w},
$$

где $P_{N_{c}}(\lambda)$ - полином степени $N_{c}$,

$$
P_{N_{c}}(\lambda)=\lambda^{N_{c}}+s_{1} \lambda^{N_{c}-1}+s_{2} \lambda^{N_{c}-2}+\cdots
$$

\footnotetext{
6) Заметим, что мы рассматриваем периодическую задачу для цепочки Тоды, в которой только функция Бейкера-Ахиезера может приобретать нетривиальный множитель при сдвиге на период, тогда как координаты и импульсы частиц периодичны. Квазипериодичность координат и импульсов частиц (когда при сдвиге на период к ним могут добавляться некоторые константы) отвечает изменению константы связи в гамильтонианах цепочки Тоды.
} 
Спектральное уравнение зависит только от взаимно коммутирующих комбинаций динамических переменных - гамильтонианов или переменных действия, параметризующих подпространство в пространстве модулей комплексных структур гиперэллиптических кривых $\Sigma^{T C}$ рода $N_{c}-1=\operatorname{rank} S U\left(N_{c}\right)$.

Альтернативное описание той же системы возникает, если явно (до того как накладываются периодические граничные условия) решить вспомогательную линейную задачу (31), являюшуюся разностным уравнением второго порядка, которое решается простым переписыванием его в виде

$$
\psi_{i+1}=\left(\lambda-p_{i}\right) \psi_{i}-\exp \left\{\phi_{i}-\frac{\phi_{i+1}+\phi_{i-1}}{2}\right\} \psi_{i-1}
$$

В самом деле, т.к. разностное уравнение второго порядка имеет два независимых решения ${ }^{7)}$ (обозначенных $\psi^{+}$и $\psi^{-}$в формуле $(31)$ ), его естественно переписать в виде $\tilde{\psi}_{i+1}=L_{i}^{T C}(\lambda) \tilde{\psi}_{i}$, где $\tilde{\psi}_{i}$ - двумерные векторы (столбцы), а $L_{i}^{T C}$ - цепочка "матриц Лакса" $2 \times 2$ [33], имеющих (после простого “калибровочного преобразования") вид

$$
L_{i}^{T C}(\lambda)=\left(\begin{array}{cc}
p_{i}+\lambda & e^{\phi_{i}} \\
e^{-\phi_{i}} & 0
\end{array}\right), \quad i=1, \ldots, N_{c}
$$

Это представление цепочки Тоды удобно для исследования гамильтоновыми методами [33]: матришы (40) удовлетворяют квадратичному пуассонову соотношению $r$-матричного типа [34] (эквивалентному каноническим соотношениям $\left\{p_{i}, \phi_{j}\right\}=\delta_{i j}$ )

$$
\left\{L_{i}^{T C}(\lambda) \stackrel{\otimes}{,} L_{j}^{T C}\left(\lambda^{\prime}\right)\right\}=\delta_{i j}\left[r\left(\lambda-\lambda^{\prime}\right), L_{i}^{T C}(\lambda) \otimes L_{j}^{T C}\left(\lambda^{\prime}\right)\right]
$$

с (не зависяшей от номера $i$ !) числовой рациональной $r$-матрицей, удовлетворяющей классическому уравнению Янга-Бакстера $r(\lambda)=\frac{1}{\lambda} \sum_{a=1}^{3} \sigma_{a} \otimes \sigma^{a}$. Как следствие матрица монодромии

$$
T_{N_{c}}(\lambda)=\prod_{N_{c} \geq i \geq 1}^{\curvearrowleft} L_{i}(\lambda)
$$

удовлетворяет тому же соотношению

$$
\left\{T_{N_{c}}(\lambda) \stackrel{\otimes}{,} T_{N_{c}}\left(\lambda^{\prime}\right)\right\}=\left[r\left(\lambda-\lambda^{\prime}\right), T_{N_{c}}(\lambda) \otimes T_{N_{c}}\left(\lambda^{\prime}\right)\right]
$$

а интегралы движения цепочки Тоды генерируются спектральным уравнением в другой форме:

$$
\underset{2 \times 2}{\operatorname{det}}\left(T_{N_{c}}^{T C}(\lambda)-w\right)=w^{2}-w \operatorname{Tr} T_{N_{c}}^{T C}(\lambda)+\operatorname{det} T_{N_{c}}^{T C}(\lambda)=w^{2}-w \operatorname{Tr} T_{N_{c}}^{T C}(\lambda)+1=0
$$

или

$$
\mathcal{P}(\lambda, w)=w+\frac{1}{w}-\operatorname{Tr} T_{N_{c}}^{T C}(\lambda)=w+\frac{1}{w}-2 P_{N_{c}}(\lambda)=0
$$

\footnotetext{
${ }^{7)}$ Начальные условия к рекурсионному соотношению (39) задаются двумя произвольными функциями, например $\psi_{1}$ и $\psi_{2}$.
} 
(здесь использовано, что $\operatorname{det}_{2 \times 2} L^{T C}(\lambda)=1$ приводит к $\operatorname{det}_{2 \times 2} T_{N_{c}}^{T C}(\lambda)=1$ ). Правая часть (45) представляет собой полином по $\lambda$ степени $N_{c}$, коэффициенты которого - интегралы движения, т.к.

$$
\begin{aligned}
\left\{\operatorname{Tr} T_{N_{c}}(\lambda), \operatorname{Tr} T_{N_{c}}\left(\lambda^{\prime}\right)\right\} & =\operatorname{Tr}\left\{T_{N_{c}}(\lambda)^{\otimes}, T_{N_{c}}\left(\lambda^{\prime}\right)\right\}= \\
& =\operatorname{Tr}\left[r\left(\lambda-\lambda^{\prime}\right), T_{N_{c}}(\lambda) \otimes T_{N_{c}}\left(\lambda^{\prime}\right)\right]=0
\end{aligned}
$$

Производящий дифференциал (21) в случае цепочки Тоды имеет вид

$$
d S^{T C}=\lambda \frac{d w}{w} .
$$

Действительно, его производные по $g$ выделенным модулям - коэффициентам полинома $P_{N_{c}}(\lambda)$ в формулах $(38),(45)$ :

$$
\left.\frac{\partial d S^{T C}}{\partial s_{k}} \equiv \frac{\partial d S^{T C}}{\partial s_{k}}\right|_{\lambda=\mathrm{const}}=\lambda d \frac{\partial}{\partial s_{k}} \log w=2 \lambda d\left(\frac{\frac{\partial P_{N_{c}}}{s_{k}}}{y}\right) \cong \frac{\lambda^{k+1} d \lambda}{y}
$$

являются (с точностью до полных производных, что обозначено $\cong$ ) голоморфными дифиференциалами на кривой $(36),(37),(45)$. При этом следует считать, что производные по модулям вычисляются при постоянном $\lambda$ (см. ниже более подробное обсуждение этого вопроса). Явные формулы для препотенциалов, отвечающих интегрируемой системе, рассмотренной в этом разделе, будут приведены в разделе 3 .

2.1. Эллиптическая деформация представления $N_{c} \times N_{c}$ : модель Калоджеро-Мозера и взаимодействие с полями материи в присоединенном представлении. $N_{c} \times N_{c}$-матричный оператор Лакса для $G L\left(N_{c}\right)$ системы Калоджеро, явно зависяший от спектрального параметра, имеет вид [35]

$$
\begin{aligned}
\mathcal{L}^{\mathrm{C}}(\xi) & \left(\mathbf{p H}+\sum_{\boldsymbol{\alpha}} F(\mathbf{q} \boldsymbol{\alpha} \mid \xi) E_{\boldsymbol{\alpha}}\right)= \\
= & \left(\begin{array}{cccc}
p_{1} & F\left(q_{1}-q_{2} \mid \xi\right) & \ldots & F\left(q_{1}-q_{N_{c}} \mid \xi\right) \\
F\left(q_{2}-q_{1} \mid \xi\right) & p_{2} & \ldots & F\left(q_{2}-q_{N_{c}} \mid \xi\right) \\
\ldots \ldots \ldots \ldots \ldots \ldots \ldots & \ldots \ldots \ldots \ldots \ldots \ldots
\end{array}\right)
\end{aligned}
$$

Его матричные элементы

$$
F(q \mid \xi)=m \frac{\sigma(q+\xi)}{\sigma(q) \sigma(\xi)} e^{\zeta(q) \xi}
$$

выражаются через эллиптические функции Вейерштрасса, т.е. оператор Лакса $\mathcal{L}(\xi)$ определен на эллиптической кривой $E(\tau)$ (комплексном торе с периодами $\omega, \omega^{\prime}$ и модулем $\left.\tau=\omega^{\prime} / \omega\right)$. Константа взаимодействия в системе Калоджеро $m^{2}$ с точки зрения четырехмерной интерпретации выражается через массу $m$ присоединенного $\mathcal{N}=2$ гипермультиплета, нарушаюшего $\mathcal{N}=4$ суперсимметрию до $\mathcal{N}=2$ [4].

Из (49) сразу следует, что спектральная кривая $\Sigma^{\mathrm{C}}$ для $G L\left(N_{c}\right)$ системы Калоджеро

$$
\operatorname{det}_{N_{c} \times N_{c}}\left(\mathcal{L}^{\mathrm{C}}(\xi)-\lambda\right)=0
$$


определяется как накрытие над эллиптической кривой $E(\tau)$

$$
y^{2}=\left(x-e_{1}\right)\left(x-e_{2}\right)\left(x-e_{3}\right)
$$

с каноническим голоморфным дифференциалом

$$
d \xi=2 \frac{d x}{y} .
$$

Массы БПЗ-состояний (26) (а и $\mathbf{a}_{D}$ ) задаются периодами производящего 1-дифференциала

$$
d S^{\mathrm{C}} \cong \lambda d \xi
$$

по нестягиваемым контурам на $\left.\Sigma^{\mathrm{C}} 8\right)$. Интегрируемость модели Калоджеро-Мозера может быть описана, например, на языке пуассоновой структуры

$$
\left\{\mathcal{L}(\xi)^{\otimes}, \mathcal{L}\left(\xi^{\prime}\right)\right\}=\left[\mathcal{R}_{12}^{\mathrm{C}}\left(\xi, \xi^{\prime}\right), \mathcal{L}(\xi) \otimes \mathbf{1}\right]-\left[\mathcal{R}_{21}^{\mathrm{C}}\left(\xi, \xi^{\prime}\right), \mathbf{1} \otimes \mathcal{L}\left(\xi^{\prime}\right)\right]
$$

определяемой динамической эллиптической $\mathcal{R}$-матрищей [36], обеспечивающей инволютивность собственных значений матрицы $\mathcal{L}$.

Периодическая цепочка Тоды получается из эллиптической модели Калоджеро в специальном двойном скейлинговом пределе [37], когда $m$ и $-i \tau$ стремятся к бесконечности, а

$$
q_{i}-q_{j}=\frac{1}{2}\left[(i-j) \log m+\left(\phi_{i}-\phi_{j}\right)\right]
$$

При этом безразмерная константа связи $\tau$ переходит в размерный параметр $\Lambda^{N_{c}} \sim$ $m^{N_{c}} e^{i \pi \tau}$. В рассматриваемом пределе эллиптическая кривая $E(\tau)$ вырождается в цилиндр с координатой $w=e^{\xi} e^{i \pi \tau}$, а производяший 1-дифференциал $d S^{\mathrm{C}}-$ в производяший дифференциал цепочки Тоды $d S^{T C}$ :

$$
d S^{\mathrm{C}} \rightarrow d S^{T C} \cong \lambda \frac{d w}{w}
$$

Оператор Лакса системы Калоджеро переходит в оператор Лакса (34):

$$
\mathcal{L}^{\mathrm{C}}(\xi) d \xi \rightarrow \mathcal{L}^{T C}(w) \frac{d w}{w}
$$

а спектральная кривая приобретает форму (35). Именно поэтому систему Калоджеро-Мозера можно рассматривать как эллиптическую деформацию цепочки Тоды. В отличие от цепочки Тоды формула (50) не можсет быть переписана в виде (36), т.е. специфическая зависимость спектрального уравнения (35) от $w$ не сохраняется при вложении цепочки Тоды в систему Калоджеро-Мозера. Однако форма (36) естественным

\footnotetext{
${ }^{8)}$ Отметим, что хотя в задаче об эллиптической системе Калоджеро-Мозера род кривой $g$, определяемой уравнением (50), равен $N_{c}$ (в то время как, вообше говоря, род кривой, задаваемой $N_{c} \times N_{c}$-матрицей, растет как $\left.N_{c}^{2}\right)$, нетривиальный смысл имеют лишь $2\left(N_{c}-1\right)$ контуров (и соответственно периодов), проекция которых на базовую кривую (51) тривиальна. Это частный случай обсуждавшихся выше многообразий Прима, когда рассматриваются только те контуры на накрытии, проекции которых на базу тривиальны.
} 
образом сохраняется при интерпретации цепочки Тоды как частного случая спиновых моделей.

В простейшем примере $N_{c}=2$ кривая $\Sigma^{\mathrm{C}}$ имеет род 2 . Действительно, в этом частном случае уравнение (50) преврашается в

$$
\mathcal{P}(\lambda, x)=\lambda^{2}-h_{2}+\frac{g^{2}}{\omega^{2}} x=\lambda^{2}-h_{2}+\frac{g^{2}}{\omega^{2}} \wp(\xi)=0 .
$$

Из (56) следует, что каждому значению $x$ отвечают две точки на кривой $\Sigma^{\mathrm{C}}$ :

$$
\lambda= \pm \sqrt{h_{2}-\frac{g^{2}}{\omega^{2}} x}
$$

т.е. $\Sigma^{\mathrm{C}}$ является двойным накрытием эллиптической кривой $E(\tau)$ с точками ветвления $x=\left(\frac{\omega}{g}\right)^{2} h_{2}$ и $x=\infty$. Поскольку сама координата $x$ является эллиптической на $E(\tau)$ (когда эллиптическая кривая рассматривается как двойное накрытие сферы $C P^{1}$ ), значение $x=\left(\frac{\omega}{g}\right)^{2} h_{2}$ отвечает паре точек на $E(\tau)$, отличаюшихся знаком $y$. Это было бы верно и для значения $x=\infty$, но $x=\infty$ - одна из точек ветвления в параметризации (51) кривой $E(\tau)$. Таким образом, два разреза между $x=\left(\frac{\omega}{g}\right)^{2} h_{2}$ и $x=\infty$ на каждом из листов $E(\tau)$ в действительности сливаются в единственный разрез между точками $\left(\left(\frac{\omega}{g}\right)^{2} h_{2},+\right)$ и $\left(\left(\frac{\omega}{g}\right)^{2} h_{2},-\right)$. Поэтому кривую $\Sigma^{\mathrm{C}}$ можно рассматривать как два тора $E(\tau)$, склеенных вдоль одного разреза, т.е. $\Sigma_{N_{c}=2}^{\mathrm{C}}$ является кривой рода 2 . Кривая $\Sigma_{N_{c}=2}^{\mathrm{C}}$ случайно оказывается гиперэллиптической (только для $N_{c}=2$ !).

В качестве двух голоморфных 1-дифференциалов на кривой (58) можно выбрать

$$
v=\frac{d x}{y} \sim \frac{\lambda d \lambda}{y}, \quad V=\frac{d x}{y \lambda} \sim \frac{d \lambda}{y},
$$

так что

$$
d S \cong \lambda d \xi=\sqrt{h_{2}-\frac{g^{2}}{\omega^{2}} \wp(\xi)} d \xi=\frac{d x}{y} \sqrt{h_{2}-\frac{g^{2}}{\omega^{2}} x} .
$$

Легко проверить, что

$$
\frac{\partial d S}{\partial h_{2}} \cong \frac{1}{2} \frac{d x}{y \lambda}
$$

Наличие в правой части лишь одного из двух голоморфных дифференциалов (60) связано с их различной четностью относительно $\mathbf{Z}_{2} \otimes \mathbf{Z}_{2}$ симметрии кривой $\Sigma^{\mathrm{C}}: y \rightarrow-y$ и $\lambda \rightarrow-\lambda$. Так как $d S$ имеет определенную четность, его периоды по двум из четырех элементарных циклов на $\Sigma^{\mathrm{C}}$ автоматически равны нулю, оставляя лишш два нетривиальных периода $\mathbf{a}$ и $\mathbf{a}_{D}$, что в точности отвечает двум независимым переменным в четырехмерной интерпретации. Более того, два ненулевых периода могут быть определены в терминах "редуцированной" кривой рода 1:

$$
Y^{2}=(y \lambda)^{2}=\left(h_{2}-\frac{g^{2}}{\omega^{2}} x\right) \prod_{a=1}^{3}\left(x-e_{a}\right),
$$


на которой

$$
d S \cong\left(h_{2}-\frac{g^{2}}{\omega^{2}} x\right) \frac{d x}{Y}
$$

Поскольку для этой кривой точка $x=\infty$ уже не является точкой ветвления, $d S$ имеет простые полюсы в $x=\infty$ (на двух разных листах $\Sigma_{\text {reduced }}^{\mathrm{C}}$ ) с вычетами $\pm \frac{g}{\omega} \sim \pm m$.

"Противоположный" предел системы Калоджеро-Мозера $g^{2} \sim m^{2} \rightarrow 0$ отвечает $\mathcal{N}=4$ суперсимметричной теории Янга-Миллса с тождественно нулевой $\beta$-функцией. Соответствующая интегрируемая система представляет собой систему свободных частищ, а производящий 1-дифференциал $d S \cong \sqrt{h_{2}} \cdot d \xi$ является просто голоморфным дифференциалом на $\left(N_{c}\right.$ копиях $) E(\tau)$.

Перейдем теперь к другой деформации цепочки Тоды, отвечающей взаимодействию $\mathcal{N}=2$ суперсимметричной калибровочной теории с полями материи в фундаментальном представлении. Согласно $[21,23]$ спектральные кривые для $\mathcal{N}=2$ суперсимметричной КХД с $N_{f}<2 N_{c}$ имеют такую же форму, как кривые, определяемые формулами (33), отличаясь лишш менее тривиальной матрицей монодромии, инварианты которой имеют вид

$$
\operatorname{Tr} T_{N_{c}}(\lambda) \equiv 2 P_{N_{c}}(\lambda)=2 P_{N_{c}}^{(0)}(\lambda)+R_{N_{c}-1}(\lambda), \quad \operatorname{det} T_{N_{c}}(\lambda)=Q_{N_{f}}(\lambda)
$$

где $Q_{N_{f}}(\lambda)$ и $R_{N_{c}-1}(\lambda)$ - некоторые (не зависяшие от $h$, но зависяшие от масс мультиплетов материи) полиномы по $\lambda$.

Естественно рассмотреть "ортогональное к предыдушему” обобщение цепочки Тоды, т.е. деформировать формулы (41)-(45), сохраняя скобки Пуассона (46):

$$
\begin{aligned}
\left\{L(\lambda)^{\otimes}, L\left(\lambda^{\prime}\right)\right\} & =\left[r\left(\lambda-\lambda^{\prime}\right), L(\lambda) \otimes L\left(\lambda^{\prime}\right)\right] \\
\left\{T_{N_{c}}(\lambda) \stackrel{\otimes}{,} T_{N_{c}}\left(\lambda^{\prime}\right)\right\} & =\left[r\left(\lambda-\lambda^{\prime}\right), T_{N_{c}}(\lambda) \otimes T_{N_{c}}\left(\lambda^{\prime}\right)\right],
\end{aligned}
$$

a, значит, и возможность построить матрицу монодромии $T(\lambda)$ путем перемножения матриц $L_{i}(\lambda)$. Таким образом, мы приходим к спектральной кривой периодической $c n u-$ новой цепочки, по-прежнему задаваемой формулой

$$
\operatorname{det}\left(T_{N_{c}}(\lambda)-w\right)=0
$$

Однако поскольку в общем случае $\operatorname{det} T_{N_{c}}(\lambda)=\prod_{i=1}^{N_{c}} \operatorname{det} L\left(\lambda-\lambda_{i}\right) \neq 1$, уравнение (66) принимает вид

$$
w+\frac{\operatorname{det} T_{N_{c}}(\lambda)}{w}=\operatorname{Tr} T_{N_{c}}(\lambda)
$$

или

$$
W+\frac{1}{W}=\frac{\operatorname{Tr} T_{N_{c}}(\lambda)}{\sqrt{\operatorname{det} T_{N_{c}}(\lambda)}} \equiv \frac{2 P_{N_{c}}(\lambda)}{\sqrt{Q_{N_{f}}(\lambda)}}
$$

а производяшая 1-форма теперь вьглядит как

$$
d S=\lambda \frac{d W}{W}
$$


Правая часть формул (67), (68) зависит от динамических переменных только через интегралы движения (которые находятся в инволюции, т.е. коммутируют друг с другом относительно скобки Пуассона). Явные примеры можно найти в работах [11, 12$]$.

В предложенной картине рациональные $(X X X)$ спиновые цепочки буквально отвечают $\mathcal{N}=2$ суперсимметричной КХД с числом мультиплетов материи $N_{f}<2 N_{c}$, в то время как конформная теория $N_{f}=2 N_{c}$ (с нулевой $\beta$-функцией) гипотетически описывается $X Y Z$-цепочкой, гамильтонова структура которой задается эллиптической алгеброй Склянина [34] (см. работу [12] по поводу детального обсуждения этого случая, в котором по-прежнему остается много открытых вопросов).

2.2. Симплектическая структура конечнозонных решений. Перейдем теперь непосредственно к обсуждению вопроса, почему и в каком смысле производящий 1-дифференциал (47) определяет интегрируемую систему. Начнем с простейшего определения, согласно которому дифференциал (47) задает симплектическую структуру на фазовом пространстве конечнозонных решений. Эта симплектическая структура была введена в [31], а в недавней работе [13] она была построена как ограничение симплектической формы интегрируемой иерархии на фазовое пространство конечнозонного решения. Мы рассмотрим более простой вывод этого утверждения $[14,15]$, справедливость которого будет подтверждена конкретными примерами, имеюшими непосредственное отношение к непертурбативным результатам в квантовых теориях, а также обсудим связь производящего дифференциала с преобразованием дуальности в непертурбативной $c<1$ теории струн $[38,39]$.

Для доказательства того, что форма (47) является производящей 1-формой для симплектической структуры, ограниченной с фазового пространства всей иерархии, рассмотрим вариацию производяшей функции

$$
S(\Sigma, \gamma)=\sum_{i} \int^{\gamma_{i}} d S=\sum_{i} \int^{\gamma_{i}} E d p
$$

где $d E$ и $d p$ (в рассмотренных выше примерах $d E=d \lambda$ и $d p=\frac{d w}{w}$ ) - два мероморфных дифференциала (с фиксированными периодами: например, для гиперэллиптической координаты $\lambda$ все $\oint d \lambda=0$ ) на кривой $\Sigma$, а $\gamma$ - дивизор решения (набор точек, в которых расположены полюсы функции Бейкера-Ахиезера). Варьируя ${ }^{9)}$, получаем

$$
\delta S=\sum_{i}(E d p)\left(\gamma_{i}\right)+\sum_{i} \int^{\gamma_{i}} \delta E d p, \quad \delta^{2} S=\delta\left(\sum_{i}(E d p)\left(\gamma_{i}\right)\right)+\sum_{i}(\delta E d p)\left(\gamma_{i}\right)
$$

Из $\delta^{2} S=0$ следует, что

$$
\varpi=\delta E \wedge \delta p=\delta\left(\sum_{i}(E d p)\left(\gamma_{i}\right)\right)=-\sum_{i}(\delta E d p)\left(\gamma_{i}\right) .
$$

\footnotetext{
9) Рассматривается полная вариация, включающая изменение модулей комплексной структуры кривой. Для того чтобы сравнивать два дифференциала на кривых с разной комплексной структурой, следует ввести связность, которая ниже будет выбрана из условия ковариантной постоянности функции $p: \delta_{\text {moduli }} p=0$ (cp. с [13], где ковариантно-постоянной функцией выбрана $E$ ).
} 
Вариацию $\delta E$ (при постоянном $p$ ) можно получить из уравнения Лакса (вспомогательной линейной задачи)

$$
\frac{\partial}{\partial t} \psi=\mathcal{L} \psi \quad(=E \psi)
$$

так что

$$
\delta E=\frac{\left\langle\psi^{\dagger} \delta \mathcal{L} \psi\right\rangle}{\left\langle\psi^{\dagger} \psi\right\rangle}
$$

и мы приходим к общей формуле

$$
\varpi=-\left\langle\delta \mathcal{L} \sum_{i}\left(d p \frac{\psi^{\dagger} \psi}{\left\langle\psi^{\dagger} \psi\right\rangle}\right)\left(\gamma_{i}\right)\right\rangle .
$$

Рассмотрим несколько важных конкретных случаев.

Уравнения КП и Кортевега-де Фриза (КдФ). В случае иерархии КП уравнение (73) имеет вид

$$
\frac{\partial}{\partial t} \psi=\left(\frac{\partial^{2}}{\partial x^{2}}+u\right) \psi \quad(=E \psi)
$$

поэтому уравнение (75) при условии $\left\langle\psi^{\dagger} \psi\right\rangle=\int_{d x} \psi^{\dagger}(x, P) \psi(x, P)$ и $\delta \mathcal{L}=\delta u(x)$ дает

$$
\varpi=-\int_{d x} \delta u(x) \sum_{i}\left(\frac{d p}{\left\langle\psi^{\dagger} \psi\right\rangle} \psi^{\dagger}(x) \psi(x)\right)\left(\gamma_{i}\right) .
$$

Появившийся дифференциал

$$
d \Omega=\frac{d p}{\left\langle\psi^{\dagger} \psi\right\rangle} \psi^{\dagger}(x) \psi(x)
$$

голоморфен всюду на $\Sigma$ за исключением “бесконечноудаленной” точки $P_{0}$, где он имеет нулевой вычет [40]. Условие равенства нулю суммы вычетов его “специальной вариации" 10)

$$
\tilde{\delta}\left(\underset{P_{0}}{\operatorname{res}} d \Omega+\sum_{i} \underset{\gamma_{i}}{\operatorname{res}} d \Omega\right)=0
$$

может быть переписано в виде

$$
\delta v(x)+\sum_{i} d \Omega\left(\gamma_{i}\right)=0
$$

где $v(x)$ - “вычет" функции Бейкера-Ахиезера в точке $P_{0}$, удовлетворяющий по определению условию $v^{\prime}(x)=u(x)$. Подставляя $(79)$ в $(75)$, получаем первую симплектическую структуру уравнения $\mathrm{K}$ д $\Phi$

$$
\varpi=\int_{d x} \delta u(x) \int_{d x^{\prime}}^{x} \delta u\left(x^{\prime}\right)
$$

\footnotetext{
${ }^{10)}$ Следует особо отметить, что "специальная вариация" $\tilde{\delta}$ отвечает ситуации, когда варьируется только $\psi$, оставляя $\psi^{\dagger}$ фиксированной.
} 
Цепочка/решетка Тоды (пример, описываюший чистую $\mathcal{N}=2$ суперсимметричную теорию Янга-Миллса). Имеем,

$$
\left\langle\psi^{\dagger} \psi\right\rangle=\sum_{n} \psi_{n}^{+}(P) \psi_{n}^{-}(P)
$$

а уравнение Лакса приобретает вид (31), где $t=t_{+}+t_{-}$, а $t_{1}=t_{+}-t_{-}-$первое время цепочки Тоды, так что

$$
\delta \lambda=\frac{\sum_{k} \psi_{k}^{+} \delta p_{k} \psi_{k}^{-}}{\left\langle\psi^{+} \psi^{-}\right\rangle}
$$

и форма (75) преврашается в

$$
\varpi=-\sum_{k} \delta p_{k} \sum_{i}\left(\frac{d p}{\left\langle\psi^{+} \psi^{-}\right\rangle} \psi_{k}^{+} \psi_{k}^{-}\right)\left(\gamma_{i}\right)
$$

Для того чтобы получить формулу

$$
\varpi=\sum_{k} \delta p_{k} \wedge \delta \phi_{k}
$$

остается доказать равенство

$$
\sum_{i}\left(\frac{d p}{\left\langle\psi^{+} \psi^{-}\right\rangle} \psi_{k}^{+} \psi_{k}^{-}\right)\left(\gamma_{i}\right)=\delta \phi_{k}
$$

Для этого вновь рассмотрим (с учетом предыдуших пояснений) специальную вариацию

$$
\tilde{\delta}\left(\underset{P_{+}}{\operatorname{res}}+\underset{P_{-}}{\operatorname{res}}+\sum_{i} \underset{\gamma_{i}}{\operatorname{res}}\right) d \Omega_{n}=0, \quad d \Omega_{n}=\frac{d p}{\left\langle\psi^{+} \psi^{-}\right\rangle} \psi_{n}^{+} \psi_{n}^{-}
$$

где первые два члена для $\psi_{n}^{ \pm} \underset{\lambda \rightarrow \lambda\left(P_{ \pm}\right)}{\sim} e^{ \pm \phi_{n}} \lambda^{ \pm n}\left(1+\mathcal{O}\left(\lambda^{-1}\right)\right)$, удовлетворяюшие "смешенным" друг относительно друга уравнениям (31) (с $\tilde{\phi}_{n}$ и $\phi_{n}$, соответственно), дают $\delta \phi_{n}=\tilde{\phi}_{n}-\phi_{n}$, а оставшаяся часть - левую часть равенства (84).

Система Калоджеро-Мозера. Вводя “стандартные” дифференциалы $d E$ и $d p$ на кривой $\Sigma(50)$ с производящей 1-формой (53), где $d p=d \xi$-голоморфный дифференциал на торе $\oint_{A} d p=\omega, \oint_{B} d p=\omega^{\prime}$, a $E=\lambda$ имеет $n-1$ полюсов с res $=1$ и один полюс с res $=-(n-1)$, функцию Бейкера-Ахиезера можно определить как решение уравнения

$$
\mathcal{L}^{\mathrm{C}}(\xi) \mathbf{a}=\lambda \mathbf{a}
$$

с сушественными особенностями [35]

$$
a_{i} \underset{E=E_{+}}{\sim} e^{x_{i} \zeta(\xi)}(1+\mathcal{O}(\xi)), \quad a_{i} \underset{E \neq E_{+}}{\sim} e^{x_{i} \zeta(\xi)}\left(-\frac{1}{n-1}+\mathcal{O}(\xi)\right)
$$


и (не зависящими от динамических переменных) полюсами $\gamma$. При этом аналогично предыдушему случаю

$$
\left\langle\psi^{\dagger} \psi\right\rangle=\sum_{i} a_{i}^{\dagger}(P) a_{i}(P), \quad \delta \mathcal{L}^{\mathrm{C}}=\frac{\sum_{i} a_{i}^{\dagger}(P) \delta p_{i} a_{i}(P)}{\sum_{i} a_{i}^{\dagger}(P) a_{i}(P)},
$$

и формула (75) преобразуется к виду

$$
\varpi=-\sum_{k} \delta p_{k} \sum_{i}\left(\frac{d \xi}{\left\langle a^{\dagger} a\right\rangle} a_{k}^{\dagger} a_{k}\right)\left(\gamma_{i}\right)
$$

условие на вычеты выглядит как

$$
\tilde{\delta}\left(\sum_{P_{j}: p=0} \underset{P_{j}}{\operatorname{res}}+\sum_{i} \underset{\gamma_{i}}{\operatorname{res}}\right) d \Omega_{k}=0, \quad d \Omega_{k}=\frac{d \xi}{\left\langle a^{\dagger} a\right\rangle} a_{k}^{\dagger} a_{k}
$$

где первая сумма берется по всем “бесконечностям" $p=0$ на каждом из листов накрытия (50). После "специальной вариации", используя (87), опять получаем

$$
\varpi=\sum_{k} \delta p_{k} \wedge \delta x_{k}
$$

Более сложные аналоги выведенных выше формул можно найти в [13]. Явный вид полученных формул является, однако, весьма нетривиальным, и поэтому, чтобы убедиться в их существовании, перейдем, наконец, к явному построению (78), (85) и (89) на примере эллиптического решения уравнения КдФ. Обозначим

$$
\psi=e^{x \zeta(z)} \frac{\sigma(x-z+\kappa)}{\sigma(x+\kappa) \sigma(z-\kappa)}, \quad \psi^{\dagger}=e^{-x \zeta(z)} \frac{\sigma(x+z+\kappa)}{\sigma(x+\kappa) \sigma(z+\kappa)}
$$

решения уравнения

$$
\left(\partial^{2}+u\right) \psi=\left(\partial^{2}-2 \wp(x+\kappa)\right) \psi=\wp(z) \psi
$$

тогда

$$
\begin{aligned}
\psi^{\dagger} \psi & =\frac{\sigma(x-z+\kappa) \sigma(x+z+\kappa)}{\sigma^{2}(x+\kappa) \sigma(z-\kappa) \sigma(z+\kappa)}=\frac{\sigma^{2}(z)}{\sigma(z+\kappa) \sigma(z-\kappa)}(\wp(z)-\wp(x+\kappa)), \\
\left\langle\psi^{\dagger} \psi\right\rangle & =\frac{\sigma^{2}(z)}{\sigma(z+\kappa) \sigma(z-\kappa)}(\wp(z)-\langle\wp(x+\kappa)\rangle),
\end{aligned}
$$

и пусть среднее по периоду $2 \widetilde{\omega}$ равно $\langle\wp(x+\kappa)\rangle=2 \tilde{\eta}$. Далее имеем

$$
\begin{aligned}
d p & =d(\zeta(z)+\log \sigma(2 \widetilde{\omega}-z+\kappa)-\log \sigma(\kappa-z))= \\
& =-d z(\wp(z)+\zeta(2 \widetilde{\omega}-z+\kappa)-\zeta(\kappa-z))=-d z(\wp(z)-2 \tilde{\eta})
\end{aligned}
$$

И

$$
\begin{gathered}
\frac{d p}{\left\langle\psi^{\dagger} \psi\right\rangle}=d z \frac{\sigma(z+\kappa) \sigma(z-\kappa)}{\sigma^{2}(z)} \\
d \Omega=\frac{d p}{\left\langle\psi^{\dagger} \psi\right\rangle} \psi^{\dagger} \psi=d z \frac{\sigma(x+z+\kappa) \sigma(x+z-\kappa)}{\sigma^{2}(z) \sigma^{2}(x+\kappa)}=d z(\wp(z)-\wp(x+\kappa)) .
\end{gathered}
$$


В данном случае “специальная вариация" $\tilde{\delta}$ имеет явный вид

$$
\begin{aligned}
\tilde{\delta} d \Omega \equiv & \frac{d p}{\left\langle\psi^{\dagger} \psi\right\rangle} \psi_{\kappa}^{\dagger} \psi_{\kappa+\delta \kappa}=d z \frac{\sigma(z+\kappa) \sigma(z-\kappa) \sigma(x+z+\kappa) \sigma(x-z+\kappa+\delta \kappa)}{\sigma^{2}(z) \sigma(x+\kappa) \sigma(z+\kappa) \sigma(x+\kappa+\delta \kappa) \sigma(z-\kappa-\delta \kappa)}= \\
= & d z \frac{\sigma(x+\kappa+z) \sigma(x+\kappa-z)}{\sigma^{2}(z) \sigma^{2}(x+\kappa)} \times \\
& \times\left[1+\delta \kappa(\zeta(x-z+\kappa)+\zeta(z-\kappa)-\zeta(x+\kappa))+\mathcal{O}\left((\delta \kappa)^{2}\right)\right]= \\
= & d z(\wp(z)-\wp(x+\kappa)) \times \\
& \times\left[1+\delta \kappa(\zeta(x-z+\kappa)+\zeta(z-\kappa)-\zeta(x+\kappa))+\mathcal{O}\left((\delta \kappa)^{2}\right)\right] .
\end{aligned}
$$

Легко заметить, что (96) имеет ненулевые вычеты в точках $z=0$ и $z=\kappa$ (полюс в точке $z=x+\kappa$ "подавлен" разностью $\wp(z)-\wp(x+\kappa))$. Эти вычеты дают

$$
\begin{aligned}
\underset{z=0}{\operatorname{res} \delta d \Omega} & \sim \delta \kappa \oint_{z \hookrightarrow 0} d z \wp(z)(\zeta(x-z+\kappa)+\zeta(z-\kappa)) \sim \\
& \sim \delta \kappa \oint_{z \hookrightarrow 0} \zeta(z) d(\zeta(x-z+\kappa)+\zeta(z-\kappa)) \sim \\
& \sim \delta \kappa(\wp(x+\kappa)+\wp(\kappa)) \sim \delta(\zeta(x+\kappa)+\zeta(\kappa)) \equiv \delta v(x)
\end{aligned}
$$

и

$$
\underset{z=\kappa}{\operatorname{res}} \delta d \Omega=\delta \kappa(\wp(\kappa)-\wp(x+\kappa))=d \Omega(\kappa),
$$

что следует из сравнения с (95). Таким образом, в данном примере существование формул (78) и (79) проверено явным вычислением.

Наконец, квантование симплектической структуры (72) в тех случаях, когда это известно, отвечает полному описанию эффективной квантовой теории. Соответствующая производящая функция $S=\sum_{k} \int^{\gamma_{k}} d S$ определяет преобразование дуальности между дуальными интегрируемыми системами (см. [38, 39]). Формулировка полной эффективной теории гипотетически должна соответствовать точному ответу для производяшей функции $\log \mathcal{T}=\log \mathcal{T}_{0}+\log \mathcal{T}_{\theta} \equiv \mathcal{F}+\log \mathcal{T}_{\theta}$, который включает также деформацию осциллируюшей части, отвечающую массивнылм возбуждениям.

2.3. Приложение: комментарии по поводу определений. Приведем теперь некоторые комментарии по поводу использованных выше формул (21) и т.п. Эти формулы, вообще говоря, нуждаются в пояснениях, т.к. в них производится дифференцирование объектов (голоморфных и мероморфных дифференциалов на комплексных кривых) по модулям, что a priori не является хорошо определенной операцией. В большинстве рассмотренных выше примеров использовались гиперэллиптические кривые (т.е. такие, для которых сушествует выделенная координата $\lambda$ или дифференциал $d \lambda$ с нулевыми периодами по любым циклам), и под производным по (“гиперэллиптическим") модулям - точкам ветвления понимались производные

$$
\left.\frac{\partial}{\partial h_{k}} d S \equiv \frac{\partial}{\partial h_{k}} d S\left(\lambda, h_{k}\right)\right|_{\lambda=\mathrm{const}},
$$

т.е. $\frac{\partial}{\partial h_{k}} d \lambda=0$. При этом возникают по меньшей мере два вопроса: 
1) почему такое определение разумно для гиперэллиптических поверхностей (например, (36), (58), (68));

2) что делать, если не существует выделенной координаты или подобного дифференциала $d \lambda$.

В данном пункте будет сделана попытка хотя бы частично ответить на эти вопросы, являюшаяся результатом обсуждений с А. Левиным и его многочисленных объяснений.

Рассмотрим определяющий интегрируемую систему в смысле работы [31] производящий дифференциал (21):

$$
\frac{\partial}{\partial h_{k}} d S \cong d v_{k},
$$

где $d v_{k}$ - некоторые голоморфные дифференциалы. Покажем сначала, что производящий дифференциал (100) может быть построен для произвольной комплексной кривой.

Рассмотрим случай голоморфных производящих дифференциалов (возникающих, например, в рамках подхода Хитчина к интегрируемым системам [41]). В этом случае обшая конструкция дифференциалов типа (100) основана на существовании голоморфных дифференциалов с $g-1$ двойнымми нулями на кривой $\Sigma_{g}$ рода $g$. Эти дифференциалы в самом общем случае имеют вид

$$
d S(P) \sim \sum_{j=1}^{g} \frac{\partial \Theta_{*}(\mathbf{0})}{\partial A_{j}} d \omega_{j}(P) \equiv H_{*}(P) \equiv \nu_{*}^{2}(P),
$$

где $\mathbf{A}(P)=\int^{P} d \boldsymbol{\omega}$ - отображение Абеля в якобиан $\operatorname{Jac}\left(\Sigma_{g}\right), \Theta$ - тэта-функция Римана $[42,43], \nu_{*}(P)$ - сечение $K^{\frac{1}{2}}$, отвечающее некоторой четной характеристике $*$ и имеющее простые нули в точках $R_{1}, \ldots, R_{g-1}$. Всего на кривой рода $g$ имеется $2^{2 g-1}+2^{g-1}$ четных и $2^{2 g-1}-2^{g-1}$ нечетных характеристик.

Определим теперь локальные координаты $\left\{h_{k}\right\}$, т.е. координаты в окрестности данной точки пространства модулей, такие что производные по соответствуюшим направлениям отвечают голоморфным дифференциалам. Это соответствие может быть установлено следующим способом:

$$
\begin{gathered}
1+\epsilon \frac{\partial}{\partial h_{k}} \leftrightarrow 1+\epsilon L_{-2}^{(k)}, \\
L_{-2}^{(k)}=\frac{1}{\xi_{k}} \frac{\partial}{\partial \xi_{k}},
\end{gathered}
$$

где $\xi_{k}$ - локальная координата на кривой в окрестности точки $R_{k}$. Очевидно, что эти операторы коммутируют

$$
\left[L_{-2}^{(j)}, L_{-2}^{(k)}\right]=0
$$

Для того чтобы проверить формулу (100), удобнее всего воспользоваться следующим базисом в пространстве голоморфных 1-дифференциалов на $\Sigma_{g}[44]$ :

$$
\begin{gathered}
d v_{k} \underset{P \rightarrow R_{k}}{\sim} d \xi_{k} \underset{P \rightarrow R_{j}}{\stackrel{+}{\rightarrow}} \delta_{j k} \xi_{j} d \xi_{j}, \\
d v_{g}=d S \underset{P \rightarrow R_{j}}{\sim} \xi_{j}^{2} d \xi, \quad j \neq k \in\{1, \ldots, g-1\} .
\end{gathered}
$$


Для дифференциалов $d v_{k}$ можно выписать явные формулы. Для этого сначала рассмотрим сечение $K^{\frac{1}{2}}$ с единственным простым полюсом в точке $R_{j}$. Такое сечение имеет вид

$$
\begin{aligned}
\psi_{j}(P) & \sim \frac{\frac{\partial}{\partial A_{i}} \Theta_{*}\left(\mathbf{A}(P)-\mathbf{A}\left(R_{j}\right)\right)}{\frac{\partial}{\partial A_{i}} \Theta_{*}(\mathbf{0}) E\left(P, R_{j}\right)}, \\
\psi_{j}(P) & \underset{P \rightarrow R_{j}}{\sim} \frac{\sqrt{d \xi_{j}}}{\xi_{j}}+\cdots,
\end{aligned}
$$

где явно использована главная форма (см., например, [42])

$$
E\left(P, P_{0}\right)=\frac{\Theta_{*}\left(\mathbf{A}(P)-\mathbf{A}\left(P_{0}\right)\right)}{\nu(P) \nu\left(P_{0}\right)} .
$$

Тогда очевидно, что

$$
d v_{k}(P)=\psi_{k}(P) \nu_{*}(P) .
$$

Теперь, чтобы проверить (100), подействуем генераторами (102) на дифференциал $d S=d v_{g}$. В результате получим

$$
\begin{aligned}
\left(1+\epsilon \frac{\partial}{\partial h_{i}}\right) d S & =\left(1+\epsilon L_{-2}^{(i)}\right) d S= \\
& =\left(1+\epsilon \frac{1}{\xi_{i}} \frac{\partial}{\partial \xi_{i}}\right)\left(\xi_{i}^{2} d \xi_{i}+\cdots\right)=\left(\xi_{i}+\frac{\epsilon}{\xi_{i}}\right)^{2} d\left(\xi_{i}+\frac{\epsilon}{\xi_{i}}\right)= \\
& =\xi_{i}^{2} d \xi_{i}+\epsilon d \xi_{i}+\mathcal{O}\left(\epsilon^{2}\right)+\cdots=d S+\epsilon d v_{k}+\mathcal{O}\left(\epsilon^{2}\right),
\end{aligned}
$$

т.е. равенство (100). Чтобы получить $2 g$-мерную интегрируемую систему, в качестве недостающего параметра можно добавить условие нормировки $d S$, т.е.

$$
d S=h_{g} d v_{g}
$$

Приведенные выше соображения почти без изменения переносятся на наиболее интересньй мероморфный случай, а явные формулы для соответствуюших дифференциалов можно извлечь из [42].

2.4. Уравнения Уизема. Перейдем теперь к другому определению производяшей 1-формы (47), тесно связанному с обшим подходом к конструкции эффективных действий, известным как метод усреднения Боголюбова-Уизема (см. [29, 45-47]). Несмотря на то что динамика Уизема описьвает коммутируюшие потоки (плоские координаты) на пространствах модулей, после усреднения по "быстрым" переменным на якобиане ее формулировка имеет наиболее простой вид в терминах дифференциалов на спектральных кривых $[45,46]$. Необходимость дальнейшего исследования производящего дифференциала вызвана прежде всего тем, что подход предыдушего пункта не позволяет естественным способом определить препотенциал $\mathcal{F}$. Динамика иерархий Уизема позволяет использовать такое определение производяшего дифференшиала (21), в результате которого возникает естественное отождествление препотенциала $\mathcal{F}$ с логарифмом $\tau$-функиии иерархии Уизема, т.е. частным случаем основного утверждения (29). 
Уравнения Уизема определяют выбор плоских координат на некотором (конечномерном, комплексном) пространстве [45], которое в интересных примерах появляется как пространство комплексных структур римановой поверхности, ассоциированной с конечнозонными решениями уравнений типа уравнений КП/Тоды. Наиболее интересные решения уравнений Уизема отвечают “модуляции” параметров конечнозонного решения интегрируемых систем типа уравнений КП/Тоды и глобальному рассмотрению связанных с ними комплексных кривых старшего рода. Например, $\tau$-функция иерархии КП, построенная по заданной спектральной кривой $\Sigma_{g}$, имеет вид

$$
\mathcal{T}\left\{t_{i}\right\}=\exp \left\{\sum t_{i} \gamma_{i j} t_{j}\right\} \Theta\left(\boldsymbol{\Phi}_{0}+\sum t_{i} \mathbf{k}_{i}\right), \quad \mathbf{k}_{i}=\oint_{\mathbf{B}} d \Omega_{i}
$$

где $\Theta$ - тэта-функция Римана $[42,43]$, построенная по якобиану $\operatorname{Jac}\left(\Sigma_{g}\right)$, а $d \Omega_{i}$ - мероморфные 1 -дифференциалы с полюсами $(i+1)$-го порядка в отмеченной точке $P_{0}$ на кривой $\Sigma_{g}$. Они полностью определяются условиями нормировки

$$
\oint_{\mathbf{A}} d \Omega_{i}=0
$$

и асимптотическим поведением

$$
d \Omega_{i}=\left(\xi^{-i-1}+o(\xi)\right) d \xi
$$

где $\xi$ - локальная координата в окрестности $P_{0}$. Модули $\left\{u_{\alpha}\right\}$ спектральной кривой являются инвариантами потоков иерархии КП

$$
\frac{\partial u_{\alpha}}{\partial t_{i}}=0
$$

Зависимость модулей от времен $t_{i}$ появляется, однако, после “модуляции" и определяется уравнениями Уизема, которые при специальном выборе координаты могут быть записаны в следуюшем наиболее простом виде:

$$
\frac{\partial d \Omega_{i}(z)}{\partial t_{j}}=\frac{\partial d \Omega_{j}(z)}{\partial t_{i}}
$$

где подразумевается, что

$$
d \Omega_{i}(z)=\frac{\partial d S(z)}{\partial t_{i}}
$$

Собственно уравнения на модули, следующие из (114), имеют вид

$$
\frac{\partial u_{\alpha}}{\partial t_{i}}=v_{i j}^{\alpha \beta}(u) \frac{\partial u_{\beta}}{\partial t_{j}}
$$

с некоторыми (в общем случае сложными) функциями $v_{i j}^{\alpha \beta}$.

С точки зрения п. 2.3 уравнения Уизема связывают две различные системы (плоских) координат на пространстве модулей. Первый набор плоских координат генерируется “усредненными” потоками иерархии КП/Тоды и отвечает действию операторов 
$L_{-n}^{0}-$ сингулярных вирасоровских генераторов в отмеченной точке $P_{0}$, соответствующей с геометрической точки зрения иерархии КП. Другой набор координат индуцируется действием операторов $L_{-2}^{\alpha}$ в специальных точках $R_{\alpha}$ на кривой $\Sigma$, которые в гиперэллиптическом случае являются точками ветвления или римановыми инвариантами.

В случае иерархии КдФ и цепочки Тоды спектральные кривые являются гиперэллиптическими. Кроме того, в случае уравнения КдФ индекс $i$ принимает только нечетные значения $i=2 j+1$, так что

$$
d \Omega_{2 j+1}(z)=\frac{\mathcal{P}_{j+g}(z)}{y(z)} d z
$$

где коэффициенты полиномов $\mathcal{P}_{j}$ фиксируются условиями нормировки (111), (112) (обычно выбирается $z\left(P_{0}\right)=\infty$ и локальный параметр в окрестности этой точки $\left.\xi=z^{-1 / 2}\right)$. В этом случае уравнения (116) могут быть диагонализованы, если в качестве координат на пространстве модулей взять $\left\{u_{\alpha}\right\}$ - координаты точек ветвления:

$$
v_{i j}^{\alpha \beta}(u)=\left.\delta^{\alpha \beta} \frac{d \Omega_{i}(z)}{d \Omega_{j}(z)}\right|_{z=u_{\alpha}} .
$$

Наконец, дифференциал $d S(z)$ (115) может быть построен для любого конечнозонного решения [31], и он совпадает с производящим дифференциалом (47). При этом равенство

$$
\frac{\partial \mathcal{F}}{\partial \mathbf{a}}=\mathbf{a}_{D}, \quad \mathbf{a}=\oint_{\mathbf{A}} d S, \quad \mathbf{a}_{D}=\oint_{\mathbf{B}} d S
$$

определяет $\tau$-функцию иерархии Уизема $\mathcal{F}=\log \mathcal{T}_{\mathrm{W}}[45,46]$. Точный ответ для производящей функции $\log \mathcal{T}=\log \mathcal{T}_{0}+\log \mathcal{T}_{\theta} \equiv \mathcal{F}+\log \mathcal{T}_{\theta}$ должен также включать деформацию осциллирующей части, отвечающую массивным возбуждениям. Ниже мы рассмотрим явный пример решений уравнений Уизема $[1,9]$.

Покажем теперь, что решения уравнений Уизема, связанные с римановыми поверхностями старших родов (уже в эллиптическом случае), приводят к непертурбативному описанию менее тривиальных физических теорий. В отличие от предыдушего примера времена иерархии Уизема теперь нетривиально связаны с модулями кривых. Эллиптическое решение уравнения $\mathrm{Kд \Phi} \mathrm{имеет} \mathrm{вид}$

$$
U\left(t_{1}, t_{3}, \ldots \mid u\right)=\frac{\partial^{2}}{\partial t_{1}^{2}} \log \mathcal{T}\left(t_{1}, t_{3}, \ldots \mid u\right)=U_{0 \wp}\left(k_{1} t_{1}+k_{3} t_{3}+\cdots+\Phi_{0} \mid \omega, \omega^{\prime}\right)+\frac{u}{3}
$$

$$
\begin{aligned}
d p & \equiv d \Omega_{1}(z)=\frac{z-\alpha(u)}{y(z)} d z \\
d Q & \equiv d \Omega_{3}(z)=\frac{z^{2}-\frac{1}{2} u z-\beta(u)}{y(z)} d z
\end{aligned}
$$

Условия нормировки (111) задают

$$
\alpha(u)=\frac{\oint_{A} \frac{z d z}{y(z)}}{\oint_{A} \frac{d z}{y(z)}}, \quad \beta(u)=\frac{\oint_{A} \frac{\left(z^{2}-\frac{1}{2} u z\right) d z}{y(z)}}{\oint_{A} \frac{d z}{y(z)}} .
$$


Простейшим примером уиземовских решений, ассоциированных с эллиптической кривой, является первое решение Гуревича-Питаевского (ГП) [48], отвечаюшее кривой специального вида

$$
y^{2}=\left(z^{2}-1\right)(z-u)
$$

с дополнительным условием, требующим неподвижности всех точек ветвления, кроме $z=u$, относительно уиземовской деформации. Нетрудно видеть, что заменой переменных $z=u+\lambda^{2}$ и $y \rightarrow y \lambda$ кривая (123) приводится к виду (частного $N_{c}=2$ случая) тодовских кривых $(37) y^{2}=\left(\lambda^{2}+u\right)^{2}-1$ с $P_{N_{c}=2}(\lambda)=\lambda^{2}+h_{2}$, т.е. $h_{2} \equiv u$. Производящий дифференциал (47), (115), отвечающий кривой (123), есть [1]

$$
d S(z)=\left(t_{1}+t_{3}\left(z+\frac{1}{2} u\right)+\cdots\right) \frac{z-u}{y(z)} d z \underset{\left\{t_{k>1}=0\right\}}{=} t_{1} \frac{z-u}{y(z)} d z .
$$

Этот дифференциал порождает решение (114) следующим образом. Дифференцируя (124), получаем

$$
\begin{aligned}
& \frac{\partial d S(z)}{\partial t_{1}}=\left(z-u-\left(\frac{1}{2} t_{1}+u t_{3}\right) \frac{\partial u}{\partial t_{1}}\right) \frac{d z}{y(z)} \\
& \frac{\partial d S(z)}{\partial t_{3}}=\left(z^{2}-\frac{1}{2} u z-\frac{1}{2} u^{2}-\left(\frac{1}{2} t_{1}+u t_{3}\right) \frac{\partial u}{\partial t_{3}}\right) \frac{d z}{y(z)}, \ldots
\end{aligned}
$$

и, сравнивая этот результат с явными формулами (121), находим

$$
\begin{aligned}
& \left(\frac{1}{2} t_{1}+u t_{3}\right) \frac{\partial u}{\partial t_{1}}=\alpha(u)-u, \\
& \left(\frac{1}{2} t_{1}+u t_{3}\right) \frac{\partial u}{\partial t_{3}}=\beta(u)-\frac{1}{2} u^{2} .
\end{aligned}
$$

Другими словами, рассматриваемая конструкция непосредственно приводит к первому решению ГП уравнений Уизема

$$
\frac{\partial u}{\partial t_{3}}=v_{31}(u) \frac{\partial u}{\partial t_{1}}
$$

где функция

$$
v_{31}(u)=\frac{\beta(u)-\frac{1}{2} u^{2}}{\alpha(u)-u}=\left.\frac{d \Omega_{3}(z)}{d \Omega_{1}(z)}\right|_{z=u}
$$

может быть выражена через эллиптические интегралы [48]. Более подробное рассмотрение можно найти в [9].

\section{3. ПРЕПОТЕНЦИАЛ НЕПЕРТУРБАТИВНЫХ РЕШЕНИЙ}

В предыдушем разделе были рассмотрены иерархии уравнений Уизема, позволяюшие в приншипе написать интегрируемые дифференциальные уравнения на производящие функции точных непертурбативных решений квантовых теорий. Ниже мы перейдем к так называемым уравнениям ассоциативности [16-18], существование которых для класса рассматриваемых непертурбативных решений можно считать следствием уравнений иерархии Уизема (хотя, вообше говоря, уравнения ассоциативности сушествуют 
для более широкого класса задач $[49,50])$. Точнее, в данном разделе будет сформулирована наиболее обшая форма уравнений ассоциативности и проверено их существование для $2 d$-топологических теорий и эффективных теорий Виттена-Зайберга. Тем самым будут явно написаны и исследованы дифференциальные уравнения, которым удовлетворяет препотенциал $\mathcal{F}$.

3.1. Уравнения ассоциативности. Препотенциал $\mathcal{F}[20,21]$ определяется в терминах семейства комплексных кривых с производящим мероморфным дифференциалом $d S$. Для калибровочной групп $G=S U\left(N_{c}\right)$ семейство $[20-22,1]$ описывается формулой (36), а производяший дифференциал - формулой (47). Препотенциал $\mathcal{F}\left(a_{i}\right)$ неявно определяется из системы уравнений (119). Согласно [1] и предыдушему разделу это определение отождествляет $\mathcal{F}\left(a_{i}\right)$ с логарифмом (редуцированной на конечномерное подпространство) $\tau$-функции интегрируемой иерархии Уизема. Сушествующий опыт работы с иерархиями Уизема $[45,49]$ подсказывает, что препотенциал $\mathcal{F}\left(a_{i}\right)$ должен удовлетворять некоторым уравнениям ассоциативности или уравнениям типа уравнений Виттена-Дийкграафа-Верлинде-Верлинде (ВДВВ) [51]. В этом разделе будет доказано, что уравнения ВДВВ для препотенциала Виттена-Зайберга имеют вид [16]

$$
\mathcal{F}_{i} \mathcal{F}_{k}^{-1} \mathcal{F}_{j}=\mathcal{F}_{j} \mathcal{F}_{k}^{-1} \mathcal{F}_{i} \quad \forall i, \quad j, k=1, \ldots, N-1
$$

где $\mathcal{F}_{i}$ обозначает (симметричную) матрищу

$$
\left(\mathcal{F}_{i}\right)_{m n}=\frac{\partial^{3} \mathcal{F}}{\partial a_{i} \partial a_{m} \partial a_{n}} .
$$

С делаем несколько замечаний по поводу уравнений (129):

1. Напомним, во-первых, что стандартные уравнения ВДВВ в $2 d$-топологических теориях поля имеют смысл ассоциативности алгебры $\phi_{i} \phi_{j}=C_{i j}^{k} \phi_{k}$ (для симметричных по $i$ и $j$ структурных констант): $\left(\phi_{i} \phi_{j}\right) \phi_{k}=\phi_{i}\left(\phi_{j} \phi_{k}\right)$ или $C_{i} C_{j}=C_{j} C_{i}$ для матриц $\left(C_{i}\right)_{n}^{m} \equiv C_{i n}^{m}$. Эти условия нетривиальны, т.к. в топологической теории структурные константы выражаются через третьи производные одной функции - препотенциала $\mathcal{F}\left(t_{i}\right): C_{i j}^{l}=\left(\eta_{(0)}^{-1}\right)^{k l} \mathcal{F}_{i j k}$ и $\mathcal{F}_{i j k}=\frac{\partial^{3} \mathcal{F}}{\partial t_{i} \partial t_{j} \partial t_{k}}$, а метрика $\eta_{k l}^{(0)}=\mathcal{F}_{0 k l}$, где $\phi_{0}=I$ - единичньй оператор. Другими словами, стандартные уравнения ВДВВ могут быть записаны как

$$
\mathcal{F}_{i} \mathcal{F}_{0}^{-1} \mathcal{F}_{j}=\mathcal{F}_{j} \mathcal{F}_{0}^{-1} \mathcal{F}_{i}
$$

В отличие от уравнений (129), в стандартных уравнениях ВДВВ $k=0$, что отвечает выделенному единичному оператору.

С другой стороны, в эффективной теории Виттена-Зайберга не существует выделенного индекса $k$ : все аргументы $a_{i}$ препотенциала равнозначны. Таким образом, если уравнения ассоциативности существуют и в этом случае, они должны быть ричны относительно перестановки индексов $i j k$, что выполняется для системы (129). Более того, система уравнений (129) верна для топологической теории обшего вида.

2. В общей теории иерархий уравнений Уизема $[45,49]$ уравнения ВДВВ возникают также в стандартной форме (131). Здесь опять существует выделенная переменная $t_{0}=x$, являющаяся как правило, первым временем в соответствующей иерархии КП/КдФ. Более того, в отличие от топологических моделей полный набор переменных 
бесконечен. В этом контексте уравнения (129) означают, что для специальных систем (для глюодинамики Виттена-Зайберга это периодические решения иерархии цепочки Тоды, отвечающие специальным гиперэллиптическим кривым (37)) существует нетривиальная редукция квазиклассической $\tau$-функции на конечное число переменных, когда она становится функцией $(N-1=g$, где $g$ - род римановой поверхности) эквивалентных аргументов $a_{i}$ и удовлетворяет более широкой системе уравнений ВДВВ, т.е. полной системе (129).

3. Из формулы (119) ясно, что переменные $a_{i}$ определены по модулю линейных замен (т.к. можно заменить любой $A$-цикл их линейной комбинацией). Уравнения (129) обладают соответствующей “ковариантностью”, наименее тривиальной частью которой является замена $\mathcal{F}_{k}$ на $\mathcal{F}_{k}+\sum_{l} \epsilon_{l} \mathcal{F}_{l}$. При этом

$\mathcal{F}_{k}^{-1} \rightarrow\left(\mathcal{F}_{k}+\sum \epsilon_{l} \mathcal{F}_{l}\right)^{-1}=\mathcal{F}_{k}^{-1}-\sum \epsilon_{l} \mathcal{F}_{k}^{-1} \mathcal{F}_{l} \mathcal{F}_{k}^{-1}+\sum \epsilon_{l} \epsilon_{l^{\prime}} \mathcal{F}_{k}^{-1} \mathcal{F}_{l} \mathcal{F}_{k}^{-1} \mathcal{F}_{l^{\prime}} \mathcal{F}_{k}^{-1}+\cdots$

Поскольку уравнения (129) верны для любой тройки индексов одновременно, они достаточны, чтобы обеспечить равенства $\mathcal{F}_{i}\left(\mathcal{F}_{k}+\sum \epsilon_{l} \mathcal{F}_{l}\right)^{-1} \mathcal{F}_{j}=$ $\mathcal{F}_{j}\left(\mathcal{F}_{k}+\sum \epsilon_{l} \mathcal{F}_{l}\right)^{-1} \mathcal{F}_{i}$. В действительности роль $\mathcal{F}_{k}$ может выполнять препотенциал $\mathcal{F}_{d \omega}$, построенньй по любому голоморфному 1-дифференциалу $d \omega$ на римановой поверхности.

4. Метрика $\eta^{(0)}$, являющаяся третьей производной (так же как и выбранные нами метрики $\left.\eta_{m n}^{(k)} \equiv\left(\mathcal{F}_{k}\right)_{m n}\right)$ в двумерных топологических теориях, всегда плоская, что позволяет выбрать “плоские координаты", в которых $\eta^{(0)}=$ const. Иногда все метрики $\eta^{(k)}$ одновременно являются плоскими. Однако это не обязательное требование: в примере с квантовыми когомологиями $C P^{2}[50,52]$ уравнения (129) выполняются для всех $k=0,1,2$, но только $\eta^{(0)}$ плоская, в то время как $\eta^{(1)}$ и $\eta^{(2)}$ имеют ненулевые кривизны.

Уравнения (129) тривиально удовлетворяются в случаях $N=2$ и $N=3$ и становятся нетривиальным условием для $N \geq 4$.

5. Когда выполняются стандартные уравнения В ДВВ (131), полная система уравнений ассоциативности (129) автоматически верна для любого $k$ при условии, что матрица $\mathcal{F}_{k}$ невырождена. Действительно ${ }^{11)}$, выражение

$$
\mathcal{F}_{i} \mathcal{F}_{k}^{-1} \mathcal{F}_{j}=\mathcal{F}_{0}\left(C_{i}^{(0)}\left(C_{k}^{(0)}\right)^{-1} C_{j}^{(0)}\right)
$$

симметрично по отношению к перестановке $i \leftrightarrow j$ при условии $\left[C_{i}^{(0)}, C_{j}^{(0)}\right]=0$.

6. Эффективная теория (119) наивно не является топологической. С четырехмерной точки зрения она описывает низкоэнергетический предел теории Янга-Миллса, который, по крайней мере, в $\mathcal{N}=2$ суперсимметричном случае нетопологический, т.к. содержит распространяющиеся безмассовые частицы. Тем не менее эта теория полностью описьвается препотенциалом, который, как теперь видно, обладает всеми (или почти всеми) свойствами препотенциалов топологических теорий. Таким образом с точки зрения теории струн, т.е. когда все описывается в терминах эффективных действий или классов универсальности, эффективные действия Виттена-Зайберга относятся к

\footnotetext{
11)Это простое доказательство было предложено А. Рослым.
} 
тому же классу, что и топологические теории. Это означает, что понятие топологических теорий может оказаться гораздо более широким, чем обычно предполагается: как, в частности отмечено в [1], оно может охватывать широкий класс инфракрасных пределов теорий поля и теорий струн, а не только теории, в которых корреляционные функции являются постоянными в пространстве-времени. Кроме того, наличие лишь третьих производных в (129), безусловно, указывает на струнную природу точных непертурбативных решений Виттена-Зайберга.

3.2. Доказательство уравнений ассоциативности. Начнем с того, что вспомним, как уравнения В ДВВ (131) доказываются в стандартных $2 d$-топологических теориях $[51,53]$. Рассмотрим простейший пример, когда $\phi_{i}-$ полиномы одной переменной $\lambda$. Доказательство сводится к проверке согласованности следуюших формул:

$$
\begin{gathered}
\phi_{i}(\lambda) \phi_{j}(\lambda)=C_{i j}^{k} \phi_{k}(\lambda) \bmod W^{\prime}(\lambda), \\
\mathcal{F}_{i j k}=\operatorname{res} \frac{\phi_{i} \phi_{j} \phi_{k}(\lambda)}{W^{\prime}(\lambda)} \equiv \sum_{\alpha} \frac{\phi_{i} \phi_{j} \phi_{k}\left(\lambda_{\alpha}\right)}{W^{\prime \prime}\left(\lambda_{\alpha}\right)}, \\
\eta_{k l}=\operatorname{res} \frac{\phi_{k} \phi_{l}(\lambda)}{W^{\prime}(\lambda)} \equiv \sum_{\alpha} \frac{\phi_{k} \phi_{l}\left(\lambda_{\alpha}\right)}{W^{\prime \prime}\left(\lambda_{\alpha}\right)}, \\
\mathcal{F}_{i j k}=\eta_{k l} C_{i j}^{l},
\end{gathered}
$$

где $\lambda_{\alpha}$ - корни полинома $W^{\prime}(\lambda)$.

В дополнении к условиям согласованности формул (134)-(137) следует также помнить, что даннье $\mathcal{F}_{i j k}$, входяшие в $(135)$, являются третьими производными функции $\mathcal{F}(a)$, т.e.

$$
\mathcal{F}_{i j k}=\frac{\partial^{3} \mathcal{F}}{\partial a_{i} \partial a_{j} \partial a_{k}} .
$$

Свойство интегрируемости (135) следует из отдельных аргументов и может быть проверено независимо. Однако если заданы (134)-(136), доказательство (137) почти очевидно:

$$
\eta_{k l} C_{i j}^{l}=\sum_{\alpha} \frac{\phi_{k} \phi_{l}\left(\lambda_{\alpha}\right)}{W^{\prime \prime}\left(\lambda_{\alpha}\right)} C_{i j}^{l}=\sum_{\alpha} \frac{\phi_{k}\left(\lambda_{\alpha}\right)}{W^{\prime \prime}\left(\lambda_{\alpha}\right)} \phi_{i}\left(\lambda_{\alpha}\right) \phi_{j}\left(\lambda_{\alpha}\right)=\mathcal{F}_{i j k} .
$$

Заметим, что (134) определено по модулю $W^{\prime}(\lambda)$, но $W^{\prime}\left(\lambda_{\alpha}\right)=0$ во всех точках $\lambda_{\alpha}$. Теперь предположим, что изменилось определение метрики:

$$
\eta_{k l} \rightarrow \eta_{k l}(\omega)=\sum_{\alpha} \frac{\phi_{k} \phi_{l}\left(\lambda_{\alpha}\right)}{W^{\prime \prime}\left(\lambda_{\alpha}\right)} \omega\left(\lambda_{\alpha}\right) .
$$

Тогда уравнения В ДВВ будут по-прежнему верными, если изменить определение алгебры (134) на

$$
\phi_{i}(\lambda) \phi_{j}(\lambda)=C_{i j}^{k}(\omega) \phi_{k}(\lambda) \omega(\lambda) \bmod W^{\prime}(\lambda) .
$$

Это равенство описывает ассоциативную алгебру всегда, когда полиномы $\omega(\lambda)$ и $W^{\prime}(\lambda)$ взаимно простые, т.е. не имеют обших делителей. Заметим, что формула $\mathcal{F}_{i j k}(135)$ остается справедливой, а значит, и факт, что $\mathcal{F}_{i j k}$ являются третьими производными 
той же самой функции $\mathcal{F}$, остался неизменным! Теперь можно взять за $\omega(\lambda)$ любой из операторов $\phi_{k}(\lambda)$, воспроизводя уравнения $(129)$ для любой топологической теории ${ }^{12)}$.

В случае глюодинамики Виттена-Зайберга вместо полиномов $\phi_{i}(\lambda)$ следует рассматривать канонические голоморфные дифференциалы $d \omega_{i}(\lambda)$ на гиперэллиптической кривой (36), которая может быть записана в стандартной гиперэллиптической форме (37).

Вместо (134) и (141) положим

$$
d \omega_{i}(\lambda) d \omega_{j}(\lambda)=C_{i j}^{k}(d \omega) d \omega_{k}(\lambda) d \omega(\lambda) \bmod \frac{d P_{N}(\lambda) d \lambda}{y^{2}}
$$

В отличие от (141) теперь невозможно просто выбрать $\omega=1$ (чтобы воспроизвести (134), необходимо взять какой-нибудь 1-дифференциал). Возьмем $d \omega$ в виде голоморфного 1-дифференциала. Однако поскольку не существует выделенного голоморфного дифференциала, а имеется их $g$-параметрическое семейство, в качестве $d \omega$ можно взять любой из этих дифференциалов. Единственное требование - взаимная простота по отношению к выбранным дифференциалам $d P_{N}(\lambda) / y$.

Если алгебра (142) существует, то структурные константы $C_{i j}^{k}(d \omega)$ удовлетворяют условию ассоциативности (при взаимной простоте $d \omega$ и $\left.d P_{N} / y\right)$. Таким образом, остается доказать, что алгебра действительно существует, т.е. что при заданном $d \omega$ можно найти (не зависяшие от $\lambda$ ) $C_{i j}^{k}$. Это легко проверить, вспомнив, что все $d \omega_{i}$ суть линейные комбинации:

$$
\begin{gathered}
d v_{k}(\lambda)=\frac{\lambda^{k-1} d \lambda}{y}, \quad k=1, \ldots, g \\
d v_{k}(\lambda)=\sigma_{k i} d \omega_{i}(\lambda), \quad d \omega_{i}=\left(\sigma^{-1}\right)_{i k} d v_{k}, \quad \sigma_{k i}=\oint_{A_{i}} d v_{k}
\end{gathered}
$$

а также $d \omega(\lambda)=s_{k} d v_{k}(\lambda)$. Тогда уравнение (142) является в действительности соотношением между полиномами

$$
\left(\sigma_{i i^{\prime}}^{-1} \lambda^{i^{\prime}-1}\right)\left(\sigma_{j j^{\prime}}^{-1} \lambda^{j^{\prime}-1}\right)=C_{i j}^{k}\left(\sigma_{k k^{\prime}}^{-1} \lambda^{k^{\prime}-1}\right)\left(s_{l} \lambda^{l-1}\right)+p_{i j}(\lambda) P_{N}^{\prime}(\lambda)
$$

В левой части стоит полином степени $2(g-1)$. Так как $P_{N}^{\prime}(\lambda)$ - полином степени $N-1=g, p_{i j}(\lambda)$ должен быть полиномом степени $2(g-1)-g=g-2$. Равенство двух полиномов степени $2(g-1)$ налагает $2 g-1$ условий на коэффициенты. Имеется свобода в выборе $C_{i j}^{k}$ и $p_{i j}(\lambda)$ (с фиксированными $i, j$ ), т.е. имеется $g+(g-1)=2 g-1$ свободных параметров: ровно столько сколько необходимо. Возникаюшая система линейных уравнений невырождена при взаимно простых $d \omega$ и $d P_{N} / y$.

Таким образом, мы доказали, что алгебра (142) существует (при заданном $d \omega$ ), a значит, структурные константы $C_{i j}^{k}(d \omega)$ удовлетворяют условию ассоциативности

$$
C_{i}(d \omega) C_{j}(d \omega)=C_{j}(d \omega) C_{i}(d \omega)
$$

\footnotetext{
12) Чтобы уравнения (129) имели смысл, следует позаботиться о том, чтобы полином $W^{\prime}(\lambda)$ имел только простые нули, в противном случае некоторые матрицы $\mathcal{F}_{k}$ могут оказаться вырожденными и необратимыми.
} 
Наконец, вместо (135) имеем [45, 49]:

$$
\begin{aligned}
\mathcal{F}_{i j k} & =\frac{\partial^{3} \mathcal{F}}{\partial a_{i} \partial a_{j} \partial a_{k}}=\frac{\partial T_{i j}}{\partial a_{k}}=\operatorname{res}_{d \lambda=0} \frac{d \omega_{i} d \omega_{j} d \omega_{k}}{d \lambda\left(\frac{d w}{w}\right)}= \\
& =\operatorname{res}_{d \lambda=0} \frac{d \omega_{i} d \omega_{j} d \omega_{k}}{d \lambda \frac{d P_{N}}{y}}=\sum_{\alpha} \frac{\widehat{\omega}_{i}\left(\lambda_{\alpha}\right) \widehat{\omega}_{j}\left(\lambda_{\alpha}\right) \widehat{\omega}_{k}\left(\lambda_{\alpha}\right)}{P_{N}^{\prime}\left(\lambda_{\alpha}\right) / \hat{y}\left(\lambda_{\alpha}\right)} .
\end{aligned}
$$

Сумма в правой части берется по всем $2 g+2$ точкам ветвления $\lambda_{\alpha}$ гиперэллиптической кривой (т.е. по нулям $\left.y^{2}=P_{N}^{2}(\lambda)-1=\prod_{\alpha=1}^{N}\left(\lambda-\lambda_{\alpha}\right)\right) ; d \omega_{i}(\lambda)=\left(\widehat{\omega}_{i}\left(\lambda_{\alpha}\right)+\right.$ $\left.\mathcal{O}\left(\lambda-\lambda_{\alpha}\right)\right) d \lambda, \hat{y}^{2}\left(\lambda_{\alpha}\right)=\prod_{\beta \neq \alpha}\left(\lambda_{\alpha}-\lambda_{\beta}\right)$. Формулу (146) легко написать, пользуясь результатами работы [45], ее доказательство можно найти, например, в [16].

Определим метрику следующим образом:

$$
\eta_{k l}(d \omega)=\operatorname{res}_{d \lambda=0} \frac{d \omega_{k} d \omega_{l} d \omega}{d \lambda\left(\frac{d w}{w}\right)}=\underset{d \lambda=0}{\operatorname{res}} \frac{d \omega_{k} d \omega_{l} d \omega_{k}}{d \lambda \frac{d P_{N}}{y}}=\sum_{\alpha} \frac{\widehat{\omega}_{k}\left(\lambda_{\alpha}\right) \widehat{\omega}_{l}\left(\lambda_{\alpha}\right) \widehat{\omega}\left(\lambda_{\alpha}\right)}{P_{N}^{\prime}\left(\lambda_{\alpha}\right) / \hat{y}\left(\lambda_{\alpha}\right)}
$$

В частности, выбирая $d \omega=d \omega_{k}, \eta_{i j}\left(d \omega_{k}\right)=\mathcal{F}_{i j k}$, приходим к уравнениям (129). С помошью (142), (146) и (147) можно проверить, что

$$
\mathcal{F}_{i j k}=\eta_{k l}(d \omega) C_{i j}^{k}(d \omega)
$$

Заметим, что $\mathcal{F}_{i j k}=\frac{\partial^{3} \mathcal{F}}{\partial a_{i} \partial a_{j} \partial a_{k}}$ в левой части $(148)$ не зависит от $d \omega !$ Правая часть $(148)$ равна

$$
\begin{aligned}
\eta_{k l}(d \omega) C_{i j}^{k}(d \omega) & =\underset{d \lambda=0}{\operatorname{res}} \frac{d \omega_{k} d \omega_{l} d \omega}{d \lambda\left(\frac{d w}{w}\right)} C_{i j}^{l}(d \omega)=\underset{d \lambda=0}{\operatorname{res}} \frac{d \omega_{k}}{d \lambda\left(\frac{d w}{w}\right)}\left(d \omega_{i} d \omega_{j}-p_{i j} \frac{d P_{N} d \lambda}{y^{2}}\right)= \\
& =\mathcal{F}_{i j k}-\underset{d \lambda=0}{\operatorname{res}} \frac{d \omega_{k}}{d \lambda\left(\frac{d P_{N}}{y}\right)} p_{i j}(\lambda) \frac{d P_{N} d \lambda}{y^{2}}= \\
& =\mathcal{F}_{i j k}-\underset{d \lambda=0}{\operatorname{res}} \frac{p_{i j}(\lambda) d \omega_{k}(\lambda)}{y}
\end{aligned}
$$

где второе равенство следует из (142). Остается доказать, что последний член равен нулю для любых $i, j, k$. Это вытекает из того, что $p_{i j}(\lambda) d \omega_{k}(\lambda) / y$ сингулярен лиш в нулях $y$ и несингулярен в точке, где $\lambda=\infty$, поскольку $p_{i j}(\lambda)$ - полином достаточно малой степени $g-2<g+1$. Таким образом, сумма его вычетов в точках ветвления является суммой всех его вычетов и поэтому равна нулю.

Таким образом, в данном пункте доказаны условия ассоциативности для любого голоморфного дифференциала $d \omega$. Выбирая $d \omega=d \omega_{k}$ (который, очевидно, является взаимно простым с $\left.d P_{N} / y\right)$, получаем (129).

3.3. Алгебраическая структура уравнений ассоциативности. Обратимся теперь более подробно к алгебраической структуре, лежащей в основе уравнений В ДВВ $[18,17]$. Напомним, что для любой метрики

$$
G=\sum_{m} \eta^{(m)} \mathcal{F}_{m}
$$


с помощью которой поднимаются или опускаются индексы

$$
C_{j}^{(G)}=G^{-1} \mathcal{F}_{j}
$$

т.е. $C_{j k}^{i}=\left(G^{-1}\right)^{i m} \mathcal{F}_{m j k}$ или $\mathcal{F}_{i j k}=G_{i m} C_{j k}^{m}{ }^{13)}$, уравнения ВДВВ просто означают, что матрицы $C$ коммутируют

$$
C_{i} C_{j}=C_{j} C_{i} \quad \forall i, j
$$

(а значит, могут быть диагонализованы одновременно). Формула (152) следует из (129), однако обратное неверно: уравнениями ассоциативности следует называть или (129), или систему (150), (151) и (152). Напомним также, что уравнения ассоциативности (152) эквивалентны условию ассоциативности умножения наблюдаемы $x \phi_{i}$ (например, элементов кирального кольца в $2 d$-топологических моделях [54])

$$
\begin{aligned}
\phi_{i} \circ \phi_{j} & =C_{i j}^{k} \phi_{k}, \\
\left(\phi_{i} \circ \phi_{j}\right) \circ \phi_{k} & =\phi_{i} \circ\left(\phi_{j} \circ \phi_{k}\right),
\end{aligned}
$$

при этом

$$
\mathcal{F}_{i j k}=\left\langle\left\langle\phi_{i} \phi_{j} \phi_{k}\right\rangle\right\rangle
$$

представляет собой (деформированную) 3-точку на сфере.

Основным примером алгебры (153) служит умножение полиномов по модулю $d P$ :

$$
\phi_{i}(\lambda) \phi_{j}(\lambda)=C_{i j}^{k} \phi_{k}(\lambda) G^{\prime}(\lambda) \bmod P^{\prime}(\lambda),
$$

где $P(\lambda)$ и $G(\lambda)$ - полиномы по $\lambda$ такие, что их производные взаимно просты. Алгебра полиномов (155) с очевидностью ассоциативна, т.к. является фактором ассоциативного кольца (всех полиномов) по идеалу с образуюшим соотношением $P^{\prime}(\lambda)=0$.

Другой частью конструкции В ДВВ является формула вычетов [55]

$$
\mathcal{F}_{i j k}=\underset{d P=0}{\operatorname{res}} \frac{\phi_{i}(\lambda) \phi_{j}(\lambda) \phi_{k}(\lambda)}{P^{\prime}(\lambda)} d \lambda,
$$

согласованная с (151),

$$
G^{\prime}(\lambda)=g^{(m)} \phi_{m}(\lambda), \quad G_{i j}=g^{(m)} \mathcal{F}_{i j m} .
$$

Последняя составляюшая - формула, выражающая плоские координаты на пространстве модулей $a_{i}$ через полином $P(\lambda)[45]$ :

$$
a_{i}=-\frac{N}{i(N-i)} \operatorname{res}\left(P^{\frac{i}{N}} d G\right), \quad N=\operatorname{ord}(P) .
$$

Эти формулы (уже использованные выше при доказательстве уравнений ассоциативности в чистой глюодинамики) естественным образом обобшаются на случай полиномов нескольких переменных $\phi_{i}(\vec{\lambda})=\phi_{i}\left(\lambda_{1}, \ldots, \lambda_{n}\right)$ :

$$
\phi_{i}(\vec{\lambda}) \phi_{j}(\vec{\lambda})=C_{i j}^{k} \phi_{k}(\vec{\lambda}) Q(\vec{\lambda}) \bmod \left(\frac{\partial P}{\partial \lambda_{1}}, \ldots, \frac{\partial P}{\partial \lambda_{n}}\right),
$$

\footnotetext{
${ }^{13)}$ Ниже мы не будем явно указывать метрику $(G)$ в $C^{(G)}$, а также будем подразумевать суммирование по повторяющимся индексам.
} 
а также

$$
\mathcal{F}_{i j k}=\operatorname{res}_{d P=0} \frac{\phi_{i}(\vec{\lambda}) \phi_{j}(\vec{\lambda}) \phi_{k}(\vec{\lambda})}{\prod_{\alpha=1}^{n} \frac{\partial P}{\partial \lambda_{\alpha}}} d \lambda_{1} \ldots d \lambda_{n} .
$$

Алгебра (159) всегда ассоциативна, т.к.

$$
d P=\sum_{\alpha=1}^{n} \frac{\partial P}{\partial \lambda_{\alpha}} d \lambda_{\alpha}
$$

всегда образует идеал в пространстве полиномов. Более того, в пространстве полиномов многих переменных всегда можно рассмотреть идеал общего вида $p_{1}(\vec{\lambda})=\ldots$ $\cdots=p_{n}(\vec{\lambda})=0$, где полиномы $p_{\alpha}$ взаимно просты, но не обязательно являются производными некоторого $P(\vec{\lambda})$. В этом пункте мы более подробно обсудим алгебраическую структуру, лежашую в основе уравнений В ДВВ и являюшуюся достаточно естественным обобшением (факторизованного) кольца полиномов.

Приведенное выше доказательство уравнений ассоциативности для чистой $\mathcal{N}=2$ суперсимметричной теории Янга-Миллса почти не отличалось от рассуждения в начале данного пункта, за исключением того, что вместо полиномов рассматривались (голоморфные) 1-дифференциалы на римановых поверхностях (комплексных кривых). Такие объекты всегда образуют семейство замкнутых алгебр, параметризуемых тройкой (вообще говоря, мероморфных) $d G, d \mathcal{W}, d \Lambda$. Однако подобные алгебры уже не имеют структуры колец (просто потому что произведение 1-дифференциалов уже не является 1-дифференциалом), поэтому не являются ассоциативными, хотя ассоциативность по-прежнему присутствует во многих важных случаях, например для гиперэллиптических кривых.

Алгебраическая конструкция интересна тем, что предполагает непосредственное обобщение на комплексные многообразия старших размерностей (от голоморфных 1-форм на кривых к формам на комплексных многообразиях), а это с физической точки зрения означало бы переход от уравнений на препотенциалы эффективной теории Виттена-Зайберга к гипотетическим уравнениям ассоциативности в струнных моделях.

Итак, предположим, что для некоторой теории выполняются следуюшие утверждения:

1. Сушествует замкнутая алгебра голоморфных ${ }^{14)} 1$-дифференциалов на комплексной кривой $\Sigma$ рода $g$ вида

$$
\begin{aligned}
d \omega_{i}(\lambda) d \omega_{j}(\lambda) & =C_{i j}^{k} d \omega_{k}(\lambda) d G(\lambda)+D_{i j}^{k} d \omega_{k}(\lambda) d \mathcal{W}(\lambda)+E_{i j}^{k} d \omega_{k}(\lambda) d \Lambda(\lambda)= \\
& =C_{i j}^{k} d \omega_{k}(\lambda) d G(\lambda) \bmod (d \mathcal{W}, d \Lambda)
\end{aligned}
$$

где $d \omega_{i}(\lambda), \quad i=1, \ldots, g,-$ базис в линейном пространстве $\Omega^{1}$ голоморфных 1-форм, $d G, d \mathcal{W}$ и $d \Lambda$ фиксированы, например $d G(\lambda)=\sum_{m=1}^{g} \eta^{(m)} d \omega_{m}$.

\footnotetext{
14) Поскольку кривые с отмеченными точками и соответствуюшие мероморфные диффференциалы получаются вырождением компактных комплексных кривых старшего рода, ниже мы сконцентрируем внимание лишь на "голоморфном" варианте. Напомним, что голоморфные 1-дифференциалы при вырождении приобретают полюсы 1-го порядка в отмеченных точках, квадратичные дифференциалы - полюсы 2-го порядка и т.д.
}

2 Теоретическая и математическая физика, т. 112, № 1, 1997 г. 
2. Фактор этой алгебры по соотношению $d \mathcal{W} \oplus d \Lambda \cong 0$ ассоциативен

$$
C_{i} C_{j}=C_{j} C_{i} \quad \forall i, j \quad \text { при фиксированных } \quad d G, d \mathcal{W}, d \Lambda .
$$

3. Существует формула выгетов

$$
\frac{\partial \mathcal{F}}{\partial a_{i} \partial j \partial a_{k}}=\operatorname{res}_{d \mathcal{W}=0} \frac{d \omega_{i} d \omega_{j} d \omega_{k}}{d \mathcal{W} d \Lambda}=-\underset{d \Lambda=0}{\operatorname{res}} \frac{d \omega_{i} d \omega_{j} d \omega_{k}}{d \mathcal{W} d \Lambda} .
$$

4. Существует невырожденная линейная комбинация матриц $\mathcal{F}_{i}$.

Из вышеприведенных утверждений 1-4 следует, что выполняются уравнения (129) на препотенциал $\mathcal{F}\left(a_{i}\right)$. Действительно, подставляя $(161)$ в $(163)$, имеем

$$
\mathcal{F}_{i j k}=C_{i j}^{m} G_{m k}
$$

где

$$
G_{m k}=\underset{d \mathcal{W}=0}{\operatorname{res}} \frac{d G d \omega_{m} d \omega_{k}}{d \mathcal{W} d \Lambda}=\eta^{(l)} \mathcal{F}_{l m k}
$$

а члены, пропорциональные $d \mathcal{W}$ и $d \Lambda$, в формуле (161) пропадают, т.к. сокрашаются с $d \Lambda$ или $d \mathcal{W}$ в знаменателе (163). Подставив (164) в (162), уравнения ВДВВ можно привести к виду

$$
\mathcal{F}_{i} G^{-1} \mathcal{F}_{j}=\mathcal{F}_{j} G^{-1} \mathcal{F}_{i}, \quad G=\eta^{(m)} \mathcal{F}_{m} \quad \forall\left\{\eta^{(m)}\right\}
$$

где обратимая метрика $G$ существует по условию.

Сушествование алгебры (161) довольно естественно для комплексных кривых. Действительно, на кривой рода $g$ сушествует $g$ независимых голоморфных 1-дифференциалов. Их произведения, однако, не независимы, $d \omega_{i} d \omega_{j}$ лежат в $(3 g-3)$-мерном пространстве $\Omega^{2}$ голоморфных квадратичных дифференциалов. Задав тройку голоморфных дифференциалов $d G, d \mathcal{W}$ и $d \Lambda$, получим отождествление

$$
\Omega^{1} \cdot \Omega^{1} \in \Omega^{2} \cong \Omega^{1} \cdot(d G \oplus d \mathcal{W} \oplus d \Lambda)
$$

которое, записанное в конкретном базисе, и дает (161). Для заданной пары индексов $i, j$ существует всего $3 g$ параметров $C_{i j}^{k}, D_{i j}^{k}$ и $E_{i j}^{k}$ в правой части $(161)$ с тремя "нулевыми модами" в направлениях $d G d \mathcal{W}, d G d \Lambda$ и $d \mathcal{W} d \Lambda$ (т.е. можно добавить $d \mathcal{W}$ к $C_{i j}^{k} d \omega_{k}$ и одновременно вычесть $d G$ из $\left.D_{i j}^{k} d \omega_{k}\right)$. Таким образом, получается в точности $3 g-3$ параметров, приводяших к сушествованию и единственности разложения (161).

Таким образом, замкнутость алгебры (161) не налагает никаких ограничений на вид римановой поверхности. Однако, как уже было замечено, обсуждаемая алгебра не является кольцом, т.к. рассматривается отображение $\left(\Omega^{1}\right)^{\otimes 2}$ в другое пространство: $\Omega^{1} \otimes \Omega^{1} \rightarrow \Omega^{2} \neq \Omega^{1}$. При этом фактор-алгебра (при факторизации по образующему соотношению $d \mathcal{W}=d \Lambda=0$ )

$$
d \omega_{i} \circ d \omega_{j}=C_{i j}^{k} d \omega_{k}
$$

не обязательно ассоциативна, т.е. сами по себе матрицы $C$ (без учета $D$ и $E$ ) не обязательно коммутируют. 
Ассоциативность восстанавливается, если разложение $\Omega^{3}$ (пространство голоморфных 3-дифференциалов, куда вкладывается результат тройного произведения $\left.\Omega^{1} \cdot \Omega^{1} \cdot \Omega^{1}\right)$ :

$$
\Omega^{3}=\Omega^{1} \cdot d G \cdot d G \oplus \Omega^{2} \cdot d \mathcal{W} \oplus \Omega^{2} \cdot d \Lambda,
$$

единственно. Тогда ясно, что равенство

$$
0=\left(d \omega_{i} d \omega_{j}\right) d \omega_{k}-d \omega_{i}\left(d \omega_{j} d \omega_{k}\right)=\left(C_{i j}^{l} C_{l k}^{m}-C_{i l}^{m} C_{j k}^{l}\right) d \omega_{m} d G^{2} \bmod (d \mathcal{W}, d \Lambda)
$$

приводит к $\left[C_{i}, C_{k}\right]=0$. Однако размерность пространства $\Omega^{3}$ равна $5 g-5$, в то время как число свободных параметров в правой части (169) составляет $g+2(3 g-3)=7 g-6$, кроме того, нужно учесть $g+2$ нулевых мод (лежащих в $\Omega^{1} \cdot d \mathcal{W} d \Lambda, \Omega^{1} \cdot d \mathcal{W} d G^{2}$ и $\left.\Omega^{1} \cdot d \Lambda d G^{2}\right)$. При $g>3$ имеет место неравенство $5 g-5<6 g-8$, т.е. разложение (169) неединственно, и ассоциативность может нарушаться (и нарушается), если только не существует никакой специальной причины для ее сохранения.

Такой специальной причиной может быть дополнительная симметрия кривой $\Sigma$, имеюшаяся, например, в случае кривых с инволюцией $\sigma: \Sigma \rightarrow \Sigma, \sigma^{2}=1$, такой что $\sigma\left(d \omega_{i}\right)=-d \omega_{i}$ и $\sigma(d \mathcal{W})=-d \mathcal{W}, \sigma(d \Lambda)=+d \Lambda$. В этом примере, в частности, необходимо, чтобы $d \Lambda$ отличался от $d \omega_{i}$, т.е. был бы мероморфным дифференциалом.

Частным (и наиболее известным) случаем таких кривых являются гиперэллиптические кривые

$$
Y^{2}=\operatorname{Pol}_{2 g+2}(\lambda)
$$

с инволюцией $\sigma:(Y, \lambda) \rightarrow(-Y, \lambda)$. Пространство голоморфных дифференциалов в этом случае может быть описано явно: $\Omega^{1}=\operatorname{Span}\left\{\frac{\lambda^{\alpha} d \lambda}{Y(\lambda)}\right\}, \alpha=0, \ldots, g-1$. Это пространство нечетно относительно инволюции $\sigma, \sigma\left(\Omega^{1}\right)=-\Omega^{1}$, а примером четного мероморфного 1-дифференциала может служить

$$
d \Lambda=\lambda^{r} d \lambda
$$

$\sigma(d \Lambda)=+d \Lambda$. Предположим, что $d G$ и $d \mathcal{W}$ голоморфны (т.е. элементы из $\Omega^{1}$ ) и $\sigma$-нечетны. В случае гиперэллиптических кривых с отмеченными точками $\Omega^{1}$ может также включать $\sigma$-четные 1 -дифференциалы (типа $\frac{d \lambda}{\left(\lambda-\alpha_{1}\right)\left(\lambda-\alpha_{2}\right)}$ или просто $d \Lambda$ ), в этом случае следует рассматривать алгебру (161) $\sigma$-нечетных дифференциалов $\Omega_{-}^{1}$ и считать, что $d \omega_{i}, d G$ и $d \mathcal{W}$ лежат в $\Omega_{-}^{1}$, а $d \Lambda \in \Omega_{+}^{1}$.

Пространства $\Omega^{2}$ и $\Omega^{3}$ также разлагаются в сумму $\sigma$-четных и $\sigma$-нечетных частей: $\Omega^{2}=\Omega_{+}^{2} \oplus \Omega_{-}^{2}$, а $\Omega^{3}=\Omega_{+}^{3} \oplus \Omega_{-}^{3}$. Умножение отображает $\Omega_{-}^{1}$ в пространство $\Omega_{+}^{2}$, а далее в пространство $\Omega_{-}^{3}$, имеющие размерности $2 g-1+2 n$ и $3 g-2+3 n$, соответственно, где $2 n$ - число отмеченных точек. Для наших целей удобно считать, что отмеченные точки на кривой расположены парами, где каждая точка входит со своим $\sigma$-образом. Таким образом, $n$ - это число таких $n a p$, а размерность $\Omega_{-}^{1}$ равна $g+n$.

Очевидно, что если все $d \omega_{i}$ в формуле $(161)$ принадлежат $\Omega_{-}^{1}$, то все коэффициенты $E_{i j}^{k}=0$, т.е. на самом деле разложение имеет вид

$$
\Omega_{+}^{2}=\Omega_{-}^{1} \cdot d G+\Omega_{-}^{1} \cdot d \mathcal{W}
$$


Тогда подсчет параметров дает $2 g-1+2 n=2(g+n)-1$, где -1 отвечает нулевой моде $d G d \mathcal{W}$. Таким образом, доказано существование использованной ранее гиперэллиптической редукции общей алгебры (161). Редуцированная алгебра уже ассоциативна, что следует из разложения

$$
\Omega_{-}^{3}=\Omega_{-}^{1} \cdot d G^{2}+\Omega_{+}^{2} \cdot d \mathcal{W} .
$$

Сушественно, что в рассматриваемом случае нет необходимости вообше учитывать в этом разложении $d \Lambda$, т.к. этот дифференциал не возникает в правой части соотношения (173). Подсчет параметров в разложении (174) дает $3 g-2+3 n=(g+n)+$ $(2 g-1+2 n)-1$ (где единственная нулевая мода - это $\left.d \mathcal{W} d G^{2}\right)$. Таким образом, разложение (173) единственно и алгебра ассоциативна.

$\mathrm{K}$ тому же выводу можно прийти, просто заметив, что все элементы $\Omega_{-}^{1}$ имеют вид

$$
d \omega_{i}=\frac{\phi_{i}(\lambda) d \lambda}{Y Q(\lambda)}
$$

где $\phi_{i}(\lambda)$ - полиномы, а $Q(\lambda)=\prod_{\iota=1}^{n}\left(\lambda-m_{\iota}\right)$ - некоторый новый полином, учитывающий возможные особенности в отмеченных точках $\left(m_{\iota}, \pm Y\left(m_{\iota}\right)\right)$. Тогда искомая алгебра представляет собой просто алгебру полиномов $\phi_{i}(\lambda)$, которая сушествует и ассоциативна по причинам, рассмотренным выше. Заметим также, что приведенное рассуждение легко обобшается на гиперэллиптические кривые с дополнительной инволюцией, которые появляются в подходе Виттена-Зайберга к теориям с калибровочными группами $S O(N)$ и $S p(N)$ : дополнительная инволюция в этих случаях задается отображением $\rho: \lambda \rightarrow-\lambda$. При этом в рассуждении, приведенном выше, следует заменить $\Omega_{-}^{1}$ на $\Omega_{-}^{1}$ (см. [18]).

Вернемся к формуле вычетов. Для этого рассмотрим интегрируемую систему с оператором Лакса $\mathcal{L}$, представленным в виде $N \times N$-матрицы (см. раздел 2 , где подробно разобраны примеры таких систем, имеющих отношение к непертурбативным калибровочным теориям поля, для них $N \equiv N_{c}$ ), элементы которой являются функциями на некоторой “затравочной” кривой $E$ (обычно это сфера или тор). Семейство комплексных кривых задается спектральным уравнением (ср. с формулами (35), (50))

$$
\operatorname{det}(\mathcal{L}(w)-\lambda)=0
$$

и устроено как $N$-листное разветвленное накрытие кривой $E$ :

$$
\mathcal{P}(\lambda ; w)=0,
$$

где $\mathcal{P}$ - полином по $\lambda$ степени $N$.

Интегрируемая система определяется производяшей 1-формой $d S=\Lambda d \mathcal{W}$, удовлетворяющей свойству (21), (100), т.е.

$$
\frac{\partial d S}{\partial \operatorname{moduli}} \in \Omega^{1}
$$

Структура интегрируемой системы позволяет выделить некоторое семейство голоморфных дифференциалов, которое представляется в более или менее явном виде. Пусть $s_{I}-$ координаты на пространстве модулей $\mathcal{M}$, тогда

$$
\frac{\partial d S}{\partial s_{I}} \cong \frac{\partial \Lambda}{\partial s_{I}} d \mathcal{W}=-\frac{\partial \mathcal{P}}{\partial s_{I}} \frac{d \mathcal{W}}{\mathcal{P}^{\prime}} \equiv d v_{I},
$$


и $d v_{I}$ - некоторый (не канонический) набор голоморфных дифференциалов на $\Sigma$, не обязательно тождественньй $\Omega_{-}^{1}$, т.е. он может лишь образовывать подпространство в $\Omega_{-}^{1}$ или же вообше оказаться линейно зависимым. Стандартным требованием в теории интегрируемых систем является то, что производные $d S-d v_{I}$ образуют полный базис в $\Omega_{-}^{1}$ (или в $\Omega_{--}^{1}$ ).

Препотенциал $\mathcal{F}\left(a_{I}\right)$ определяется стандартными формулами $(22),(23),(29)$ и (119). При этом циклы $A_{I}$, вообше говоря, включают $A_{i}$ - циклы окружаюшие отмеченные точки, сопряженные им незамкнутые контуры $B_{i}$ соединяют особые точки дифференциала $d S$.

Определение препотенциала $\mathcal{F}(119)$ самосогласовано, т.к. матрица периодов $\partial^{2} \mathcal{F} / \partial a_{I} \partial a_{J}$ симметрична. Действительно, продифференцируем (119) по параметрам $s_{K}$ и используем условие (179), тогда

$$
\int_{B_{I}} d v_{K}=\sum_{J} T_{I J} \oint_{A_{J}} d v_{K}
$$

где вторая производная

$$
\frac{\partial^{2} \mathcal{F}}{\partial a_{I} \partial a_{J}}=T_{I J}
$$

- матрица периодов кривой (с отмеченными точками) $\Sigma$. Как любая матрица периодов она симметрична

$$
\begin{aligned}
\sum_{I J}\left(T_{I J}-T_{J I}\right) \oint_{A_{I}} d v_{K} \oint_{A_{J}} d v_{L} & =\sum_{I}\left(\oint_{A_{I}} d v_{K} \int_{B_{I}} d v_{L}-\int_{B_{I}} d v_{K} \oint_{A_{I}} d v_{L}\right)= \\
& =\operatorname{res}\left(v_{K} d v_{L}\right)=0 .
\end{aligned}
$$

Заметим, что канонические (нормированные) голоморфные дифференциалы $d \omega_{I}$ : $\oint_{A_{I}} d \omega_{J}=\delta_{I J}$ и $\oint_{B_{I}} d \omega_{J}=T_{I J}$, отвечают координатам $a_{I}(22),(23),(119)$ на пространстве модулей.

Для вывода формулы вычетов рассмотрим производную матрицы периодов по модулям. Легко получить, что

$$
\begin{aligned}
\sum_{I J} \frac{\partial T_{I J}}{\partial s_{M}} \oint_{A_{I}} d v_{K} \oint_{A_{J}} d v_{L} & =\sum_{I}\left(\oint_{A_{I}} d v_{K} \int_{B_{I}} \frac{\partial d v_{L}}{\partial s_{M}}-\int_{B_{I}} d v_{K} \oint_{A_{I}} \frac{\partial d v_{L}}{\partial s_{M}}\right)= \\
& =\operatorname{res}\left(v_{K} \frac{\partial d v_{L}}{\partial s_{M}}\right) .
\end{aligned}
$$

Правая часть не равна нулю, т.к. дифференцирование по модулям приводит, вообше говоря, к появлению новых сингулярностей. Из формулы (179) следует, что

$$
\begin{aligned}
-\frac{\partial d v_{L}}{\partial s_{M}}= & \frac{\partial^{2} \mathcal{P}}{\partial s_{L} \partial s_{M}} \frac{d \mathcal{W}}{\mathcal{P}^{\prime}}+\left(\frac{\partial \mathcal{P}}{\partial s_{L}}\right)^{\prime}\left(-\frac{\partial \mathcal{P}}{\partial s_{M}}\right) \frac{d \mathcal{W}}{\mathcal{P}^{\prime}}-\frac{\partial \mathcal{P}}{\partial s_{L}} \frac{\partial \mathcal{P}^{\prime}}{\partial s_{M}} \frac{d \mathcal{W}}{\left(\mathcal{P}^{\prime}\right)^{2}}+ \\
& +\frac{\partial \mathcal{P}}{\partial s_{L}} \frac{\partial \mathcal{P}}{\partial s_{M}} \frac{\mathcal{P}^{\prime \prime} d \mathcal{W}}{\left(\mathcal{P}^{\prime}\right)^{3}}=\left[\left(\frac{\partial \mathcal{P} / \partial s_{L} \partial \mathcal{P} / \partial s_{M}}{\mathcal{P}^{\prime}}\right)^{\prime}+\frac{\partial^{2} \mathcal{P}}{\partial s_{L} \partial s_{M}}\right] \frac{d \mathcal{W}}{\mathcal{P}^{\prime}}
\end{aligned}
$$


и новые сингулярности (квадратичные полюсы) возникают в нулях $\mathcal{P}^{\prime}$ (т.е. в нулях $d \mathcal{W}$ ). Собирая коэффициент при ведушей сингулярности, получаем

$$
\operatorname{res} v_{K} \frac{\partial d v_{L}}{\partial s_{M}}=-\underset{d \mathcal{W}=0}{\operatorname{res}} \frac{\partial \mathcal{P}}{\partial s_{K}} \frac{\partial \mathcal{P}}{\partial s_{L}} \frac{\partial \mathcal{P}}{\partial s_{M}} \frac{d \mathcal{W}^{2}}{\left(\mathcal{P}^{\prime}\right)^{3} d \Lambda}=\underset{d \mathcal{W}=0}{\operatorname{res}} \frac{d v_{K} d v_{L} d v_{M}}{d \mathcal{W} d \Lambda}
$$

Интегралы в левой части (183) преврашают $d v_{I}$ в канонические дифференциалы $d \omega_{I}$. Та же самая матрица $\oint_{A_{I}} d v_{J}$ связывает производные по модулям $s_{I}$ с периодами $a_{I}$. Собирая все вместе, получаем (см. также [45]):

$$
\begin{aligned}
\frac{\partial T_{I J}}{\partial s^{K}} & =\operatorname{res}_{d \mathcal{W}=0} \frac{d \omega_{I} d \omega_{J} d v_{K}}{d \mathcal{W} d \Lambda}, \\
\frac{\partial^{3} F}{\partial a^{I} \partial a^{J} \partial a^{K}} & =\frac{\partial T_{I J}}{\partial a^{K}}=\operatorname{res}_{d \mathcal{W}=0} \frac{d \omega_{I} d \omega_{J} d \omega_{K}}{d \mathcal{W} d \Lambda} .
\end{aligned}
$$

Заметим, что приведенные формулы зависят лиш от симплектической структуры $d \mathcal{W} \wedge d \Lambda$, например, при инфинитезимальном сдвиге $d \mathcal{W}$ на $d \Lambda$ величина $(d \mathcal{W} d \Lambda)^{-1}$ сдвигается на $-(d \mathcal{W})^{-2}$, т.е. сдвиг не содержит полюсов в точках, где $d \Lambda=0$, а поэтому не дает вклада в формулу вычетов. Отметим, наконец, что приведенные выше рассуждения и формулы вычетов буквально применимы к системам, описываемым голоморфными дифференциалами. В случае кривых с отмеченными точками (и соответственно дифференциалов с полюсами в этих точках) приведенные вьше формулы требуют аккуратного доопределения, которое может быть найдено в [18].

\section{4. Явные примеры.}

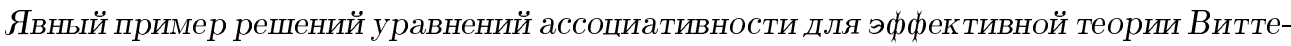
на-Зайберга. Этот пример относится к ведушему (пертурбативному) вкладу в точный препотенциал Виттена-Зайберга, который уже сам по себе удовлетворяет (129). Поскольку пертурбативный вклад выражается через элементарные функции, вычисление можно выполнить явно:

$$
\begin{aligned}
\mathcal{F}_{\text {pert }} & \equiv \mathcal{F}\left(a_{i}\right)=\left.\frac{1}{2} \sum_{\substack{m<n \\
m, n=1}}^{N}\left(A_{m}-A_{n}\right)^{2} \log \left(A_{m}-A_{n}\right)\right|_{\sum_{m} A_{m}=0}= \\
& =\frac{1}{2} \sum_{\substack{i<j \\
i, j=1}}^{N-1}\left(a_{i}-a_{j}\right)^{2} \log \left(a_{i}-a_{j}\right)+\frac{1}{2} \sum_{i=1}^{N-1} a_{i}^{2} \log a_{i} .
\end{aligned}
$$

В качестве независимых переменных можно выбрать $a_{i}=A_{i}-A_{N}$, т.к. согласно (132) система уравнений (129) ковариантна относительно таких замен.

Формула (187) означает, что в $4 d$-суперсимметричной неабелевой калибровочной теории пертурбативный вклад в эффективное действие имеет структуру

$$
\mathcal{F}_{\text {pert }}=\frac{1}{4} \sum_{\text {masses }}(\text { mass })^{2} \log (\text { mass })
$$


где в (187) все массы генерируются вакуумными средними хиггсовского поля согласно механизму спонтанного нарушения симметрии. Формулу (188) легко получить из условия на эффективный заряд

$$
\delta^{2} \mathcal{F}_{\text {pert }} \sim \sum_{\text {masses }} \log (\text { mass })
$$

который дается чисто логарифмическим вкладом одной петли.

Введем обозначения $a_{i j}=a_{i}-a_{j}$, тогда

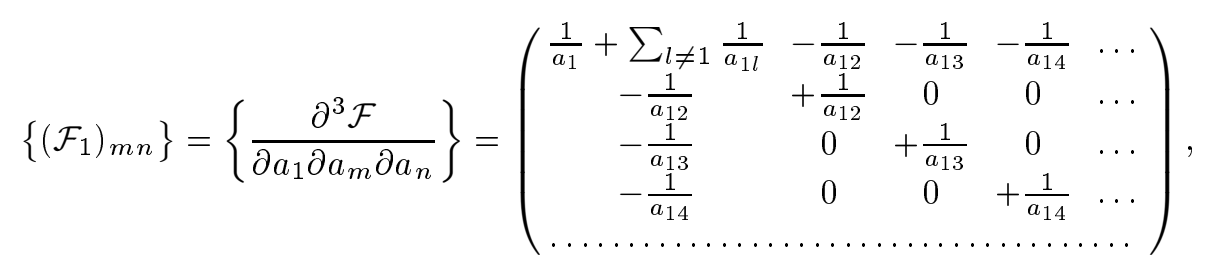

откуда следует, что матричные элементы матрицы $\mathcal{F}_{i}$ имеют вид

$$
\begin{aligned}
\left\{\left(\mathcal{F}_{i}\right)_{m n}\right\}= & \frac{\delta_{m n}\left(1-\delta_{m i}\right)\left(1-\delta_{n i}\right)}{a_{i m}}-\frac{\delta_{m i}\left(1-\delta_{n i}\right)}{a_{i n}}-\frac{\delta_{n i}\left(1-\delta_{m i}\right)}{a_{i m}}+ \\
& +\left(\frac{1}{a_{i}}+\sum_{l \neq i} \frac{1}{a_{i k}}\right) \delta_{m i} \delta_{n i} .
\end{aligned}
$$

Обратная матрица есть

$$
\left\{\left(\mathcal{F}_{k}^{-1}\right)_{m n}\right\}=a_{k}+\delta_{m n} a_{k m}\left(1-\delta_{m k}\right),
$$

например,

$$
\left\{\left(\mathcal{F}_{1}^{-1}\right)_{m n}\right\}=a_{1}\left(\begin{array}{cccc}
1 & 1 & 1 & \ldots \\
1 & 1 & 1 & \ldots \\
1 & 1 & 1 & \ldots \\
\ldots & \ldots
\end{array}\right)+\left(\begin{array}{cccc}
0 & 0 & 0 & \ldots \\
0 & a_{12} & 0 & \ldots \\
0 & 0 & a_{13} & \ldots \\
\ldots \ldots & \ldots
\end{array}\right)
$$

В качестве простейшего примера рассмотрим случай $N=4$, где достаточно проверить хотя бы одно из уравнений (129), а все остальные будут выполняться автоматически. Возьмем $k=1$, тогда

$$
\begin{gathered}
\mathcal{F}_{1}=\left(\begin{array}{ccc}
\frac{1}{a_{1}}+\frac{1}{a_{12}}+\frac{1}{a_{13}} & -\frac{1}{a_{12}} & -\frac{1}{a_{13}} \\
-\frac{1}{a_{12}} & \frac{1}{a_{12}} & 0 \\
-\frac{1}{a_{13}} & 0 & \frac{1}{a_{13}}
\end{array}\right), \quad \mathcal{F}_{2}^{-1}=\left(\begin{array}{ccc}
a_{2}+a_{21} & a_{2} & a_{2} \\
a_{2} & a_{2} & a_{2} \\
a_{2} & a_{2} & a_{2}+a_{23}
\end{array}\right), \\
\mathcal{F}_{3}=\left(\begin{array}{ccc}
\frac{1}{a_{31}} & 0 & -\frac{1}{a_{31}} \\
0 & \frac{1}{a_{32}} & -\frac{1}{a_{32}} \\
-\frac{1}{a_{31}} & -\frac{1}{a_{32}} & \frac{1}{a_{3}}+\frac{1}{a_{31}}+\frac{1}{a_{32}}
\end{array}\right),
\end{gathered}
$$

произведение $\mathcal{F}_{1} \mathcal{F}_{2}^{-1} \mathcal{F}_{3}$ принимает вид

$$
\mathcal{F}_{1} \mathcal{F}_{2}^{-1} \mathcal{F}_{3}=\left(\begin{array}{ccc}
\star & -\frac{1}{a_{13}} & \Delta+\frac{a_{21}+a_{23}}{a_{13}^{2}} \\
-\frac{1}{a_{13}} & \star & \frac{1}{a_{13}} \\
\frac{a_{21}+a_{23}}{a_{13}^{2}} & \frac{1}{a_{13}} & \star
\end{array}\right)
$$


где диагональные члены не выписаны, т.к. для доказательства (129) достаточно проверить симметричность этой матрищы. Матрица действительно симметрична, т.к.

$$
\Delta \equiv \frac{a_{2}}{a_{1} a_{3}}-\frac{a_{21}}{a_{1} a_{31}}-\frac{a_{23}}{a_{3} a_{13}}=0
$$

причем равенство $a_{i j}=a_{i}-a_{j}$ понадобилось только в этом месте.

Докажем теперь (129) в обшем случае. Проверим уравнение для обратных матриц. Именно, используя (191), (192), получим

$$
\begin{aligned}
\left(\mathcal{F}_{i}^{-1} \mathcal{F}_{j} \mathcal{F}_{k}^{-1}\right)_{\alpha \beta}=\frac{a_{i} a_{k}}{a_{j}}+\delta_{\alpha \beta}\left(1-\delta_{i \alpha}\right)\left(1-\delta_{k \alpha}\right)\left(1-\delta_{j \alpha}\right) \frac{a_{i \alpha} a_{k \beta}}{a_{j \beta}}+ \\
\quad+\delta_{j \alpha} \delta_{j \beta}\left(1-\delta_{i \alpha}\right)\left(1-\delta_{k \beta}\right)\left(\frac{1}{a_{j}}+\sum_{n \neq j} \frac{1}{a_{j n}}\right)+ \\
\quad+\delta_{j \alpha}\left(1-\delta_{i \alpha}\right) a_{i \alpha}\left(\frac{a_{k}}{a_{j}}-\frac{a_{k \beta}}{a_{j \beta}}\left(1-\delta_{k \beta}\right)\left(1-\delta_{j \beta}\right)\right)+ \\
\quad+\delta_{j \beta}\left(1-\delta_{k \beta}\right)\left(\frac{a_{i}}{a_{j}}-\frac{a_{i \alpha}}{a_{j \alpha}}\left(1-\delta_{i \alpha}\right)\left(1-\delta_{j \alpha}\right)\right)= \\
=\frac{a_{i} a_{k}}{a_{j}}+\delta_{\alpha \beta}\left(1-\delta_{i \alpha}-\delta_{k \alpha}-\delta_{j \alpha}\right) \frac{a_{i \alpha} a_{k \beta}}{a_{j \beta}}+\delta_{j \alpha} \delta_{j \beta}\left(\frac{1}{a_{j}}+\sum_{n \neq j} \frac{1}{a_{j n}}\right)+ \\
\quad+\delta_{j \alpha} a_{i \alpha}\left(\frac{a_{k}}{a_{j}}-\frac{a_{k \beta}}{a_{j \beta}}\left(1-\delta_{k \beta}-\delta_{j \beta}\right)\right)+\delta_{j \beta}\left(\frac{a_{i}}{a_{j}}-\frac{a_{i \alpha}}{a_{j \alpha}}\left(1-\delta_{i \alpha}-\delta_{j \alpha}\right)\right)
\end{aligned}
$$

где принято $i \neq j \neq k$. Первые три члена симметричны относительно перестановки $\alpha \leftrightarrow \beta$. Для доказательства симметричности последних двух членов следует использовать тождества

$$
\frac{a_{k}}{a_{j}}-\frac{a_{k \beta}}{a_{j \beta}}=\frac{a_{\beta} a_{j k}}{a_{j} a_{j \beta}} \stackrel{k=\beta}{\rightarrow} \frac{a_{k}}{a_{j}}, \quad \frac{a_{i}}{a_{j}}-\frac{a_{i \alpha}}{a_{j \alpha}}=\frac{a_{\alpha} a_{j i}}{a_{j} a_{j \alpha}} \stackrel{i=\alpha}{\rightarrow} \frac{a_{i}}{a_{j}}
$$

Тогда последняя строка в (197) равна

$$
\delta_{j \alpha}\left(1-\delta_{j \beta}\right) \frac{a_{i j} a_{j k}}{a_{j}} \frac{a_{\beta}}{a_{j \beta}}+\delta_{j \beta}\left(1-\delta_{j \alpha}\right) \frac{a_{i j} a_{j k}}{a_{j}} \frac{a_{\alpha}}{a_{j \alpha}}+\delta_{j \alpha} \delta_{j \beta} \frac{a_{k} a_{i \alpha}+a_{i} a_{k \beta}}{a_{j}} .
$$

Интересно отметить, что в данном примере $(187)$ все метрики $\eta^{(k)}$ плоские. Более того, легко найти явные плоские координаты:

$$
\begin{aligned}
\eta^{(k)} & =\eta_{i j}^{(k)} d a^{i} d a^{j}=\mathcal{F}_{i j k} d a^{i} d a_{j}=d a_{i} d a_{j} \partial_{i j}^{2}\left(\partial_{k} \mathcal{F}\right)= \\
& =\frac{d a_{k}^{2}}{a_{k}}+\sum_{l \neq k} \frac{d a_{k l}^{2}}{a_{k l}}=4\left(\left(d \sqrt{a_{k}}\right)^{2}+\sum_{l \neq k}\left(d \sqrt{a_{k l}}\right)^{2}\right)
\end{aligned}
$$

Явная вид непертурбативных вкладов (инстантоны и т.п.) в препотенциал вычисляется гораздо сложнее. Для некоторых примеров эти вклады были явно вычислены разложением точных формул типа формул Виттена-Зайберга в работе [17], обсуждение этих вкладов и их вычисление полевыми методами можно найти в работах [56]. 
Голоморфные дифференциалы на сфере с отмеченными точками. Покажем теперь, что рассмотренный пертурбативный пример отвечает рациональному вырождению спектральных кривых, а именно сфере с отмеченными точками $\lambda_{i}, i=1, \ldots, N$, где базис в пространстве $\Omega^{1}$ можно выбрать в виде

$$
d \omega_{i}=\frac{\left(\lambda_{i}-\lambda_{N}\right) d \lambda}{\left(\lambda-\lambda_{i}\right)\left(\lambda-\lambda_{N}\right)}, \quad i=1, \ldots, N-1
$$

(контуры $A_{i}$ охватывают точки $\lambda_{i}$, а сопряженные к ним контуры $B_{i}$ соединяют $\lambda_{i} \mathrm{c}$ “точкой отсчета" $\left.\lambda_{N}\right)$. Алгебра определяется как умножение $d \omega_{i}$ по модулю

$$
d \mathcal{W}=d \log P_{N}(\lambda)=\frac{d P_{N}(\lambda)}{P_{N}(\lambda)},
$$

$P_{N}(\lambda)=\prod_{i=1}^{N}\left(\lambda-\lambda_{i}\right)$, и с очевидностью является ассоциативной.

Выражения для периодов $a_{i}$ зависят от выбора производящего дифференциала $d S=\Lambda d \mathcal{W}$. С точки зрения эффективного описания Виттена-Зайберга производящий дифференциал можно выбрать двумя сушественно различными способами: $\Lambda=\lambda[1]$ и $\Lambda=\log \lambda[19]$, т.е.

$$
d S^{(4)}=\lambda d \log P_{N}(\lambda), \quad d S^{(5)}=\log \lambda d \log P_{N}(\lambda)
$$

Чтобы выполнить условие (178), нужно потребовать, чтобы $\sum_{i=1}^{N} \lambda_{i}=0$ в случае $d S^{(4)}$ и $\prod_{i=1}^{N} \lambda_{i}=1$ для случая $d S^{(5)}$. $A_{i}$-периоды тривиально вычисляются как вычеты в точках $\lambda=\lambda_{i}$, которые для $d S$ равны

$$
\begin{aligned}
& a_{i}^{(4)}=\oint_{\lambda_{i}} d S^{(4)}=\lambda_{i}, \\
& a_{i}^{(5)}=\oint_{\lambda_{i}} d S^{(5)}=\log \lambda_{i} .
\end{aligned}
$$

Соответствующие формулы вычетов имеют вид

$$
\begin{gathered}
\mathcal{F}_{i j k}^{(4)}=\sum_{m=1}^{N} \underset{\lambda_{m}}{\operatorname{res}} \frac{d \omega_{i} d \omega_{j} d \omega_{k}}{d \lambda d \log P_{N}}, \\
\mathcal{F}_{i j k}^{(5)}=\sum_{m=1}^{N} \underset{\lambda_{m}}{\operatorname{res}} \lambda \frac{d \omega_{i} d \omega_{j} d \omega_{k}}{d \lambda d \log P_{N}}, \quad i, j, k=1, \ldots, N-1,
\end{gathered}
$$

и в обоих рассматриваемых случаях мы получаем решения уравнений ВДВВ. Соответствующие препотенциалы имеют вид (187) и (см. [17, 18])

$$
\begin{gathered}
\mathcal{F}^{(5)}\left(a_{i}\right)=\sum_{1 \leq i<j \leq N} \tilde{L} i_{3}\left(e^{a_{i}-a_{j}}\right)-\frac{N}{2} \sum_{1 \leq i<j<k \leq N} a_{i} a_{j} a_{k}, \quad \sum_{i=1}^{N} a_{i}=0 \\
\partial_{x}^{2} \tilde{L} i_{3}\left(e^{x}\right) \equiv \log 2 \sinh x, \quad \tilde{L} i_{3}\left(e^{x}\right)=\frac{1}{6} x^{3}-\frac{1}{4} L i_{3}\left(e^{-2 x}\right)
\end{gathered}
$$


Они отвечают пертурбативному пределу в четырехмерных и пятимерных [19] $\mathcal{N}=2$ калибровочных теориях с группой $S U(N)$, соответственно. Заметим, однако, что формула (205) отличается от приведенной в [19] на кубичные по периодам члены, наличие которых необходимо для выполнения уравнений ассоциативности.

Если отмеченные точки $\lambda_{i}$ удовлетворяют определенным соотношениям, то практически те же формулы дают решения уравнений ВДВВ, отвечаюшие другим простым группам: $S O(N), S p(N), F_{4}$ и $E_{6,7,8}$ (группа $G_{2}$ не имеет достаточного количества параметров ( $\left.\operatorname{rank} G_{2}=2\right)$, чтобы уравнения ассоциативности в соответствуюшей калибровочной теории были нетривиальны). Согласно (68), если теперь заменить полином $P_{N}$ на

$$
P_{N} \rightarrow \frac{P_{N}}{Q_{N_{f}}^{1 / 2}}=\frac{\prod_{i=1}^{N}\left(\lambda-\lambda_{i}\right)}{\prod_{i=1}^{N_{f}}\left(\lambda-m_{i}\right)^{1 / 2}},
$$

то возникаюшие решения уравнений ассоциативности можно проинтерпретировать как пертурбативный предел $\mathcal{N}=2$ суперсимметричной КХД, т.е. калибровочной теории с полями материи в фундаментальном представлении низшей размерности. Включение взаимодействия с полями материи в других представлениях калибровочной группы, вообще говоря, разрушает уравнения ассоциативности. Заметим, что модели с произвольными представлениями не возникают естественным образом из струнной компактификации, а кроме того, для таких теорий неизвестно эффективного описания в смысле Виттена-Зайберга.

Голоморфные дифференциалы на гиперэллиптических кривых. Hепертурбативные решения и соответствуюшие препотенциалы возникают, когда каждая пара отмеченных точек "раздувается" до ручки гиперэллиптической кривой:

$$
\begin{gathered}
W+\frac{1}{W}=2 \frac{P_{N}(\lambda)}{Q(\lambda)_{N_{f}}^{1 / 2}}, \\
W-\frac{1}{W}=2 \frac{Y(\lambda)}{Q(\lambda)_{N_{f}}^{1 / 2}}, \\
Y^{2}(\lambda)=P_{N}^{2}(\lambda)-Q_{N_{f}}(\lambda) .
\end{gathered}
$$

Эти кривые вместе с производящими дифференциалами $d S$ :

$$
d S^{(4)}=\lambda \frac{d W}{W}, \quad d S^{(5)}=\log \lambda \frac{d W}{W}
$$

(т.е. $d \mathcal{W}=\frac{d W}{W}$ и $\left.d \Lambda^{(4)}=d \lambda, d \Lambda^{(5)}=\frac{d \lambda}{\lambda}\right)$, как было показано выше, отвечают интегрируемым моделям типа цепочек Тоды (см. также $[2,3,19])$. Вместе с формулой вычетов (163) они приводят к непертурбативным решениям уравнений ассоциативности (129).

Например, доказательство п. 3.2 практически без изменений переносится на случай релятивистской цепочки Тоды [57], отвечающей пятимерной $\mathcal{N}=2$ суперсимметричной чисто калибровочной теории в пространстве-времени с одним компактифицированным измерением [19]. Это происходит, в частности, потому, что спектральная кривая релятивистской цепочки Тоды практически совпадает с кривой (45) и имеет вид

$$
w+\frac{1}{w}=(\zeta \lambda)^{-N_{c} / 2} P(\lambda)
$$


т.е. также является әиперәллиптической кривой. Действительно, легко заметить, что с помощю новой переменной $Y \equiv(\zeta \lambda)^{N_{c} / 2}\left(w-\frac{1}{w}\right)$ уравнение $(209)$ принимает вид

$$
Y^{2}=P^{2}(\lambda)-4 \zeta^{2 N_{c}} \lambda^{N_{c}}
$$

где $\lambda \equiv e^{2 \xi}, \xi$ - спектральньй параметр релятивистской цепочки Тоды, а $\zeta$ - константа связи.

Тем не менее данный пример отличается от четырехмерного случая (см. п.3.2): во-первых, в случае релятивистской цепочки Тоды $s_{0} \sim \prod e^{a_{i}}=1$, а $s_{N_{c}-1}$ в отличие от обычной цепочки Тоды становится “живым” параметром; во-вторых, сушественное различие заключается в выборе производящего 1-дифференциала. Формулу (47) в пятимерном случае следует заменить на

$$
d S=\xi \frac{d w}{w} \sim \log \lambda \frac{d w}{w},
$$

так что

$$
d W_{k}=\frac{\partial d S}{\partial s_{k}} \cong \frac{\lambda^{k-1} d \lambda}{Y}, \quad k=1, \ldots, g .
$$

Несмотря на условие $s_{0}=1$, что означает отсутствие соответствуюшего дифференциала, эта формула буквально совпадает (благодаря присутствию дополнительной степени $\lambda$ в знаменателе) с формулой (143). Таким образом, релятивистской цепочке Тоды отвечает та же самая ассоциативная алгебра дифференциалов, подробно рассмотренная в п 3.2. Единственная формула, которая несколько меняется в данном случае, это формула вычетов, связываюшая дифференциалы с производными препотенциала $\mathcal{F}$,

$$
\mathcal{F}_{i j k}=\operatorname{res}_{d \lambda=0} \frac{d \omega_{i} d \omega_{j} d \omega_{k}}{\left(\frac{d \lambda}{\lambda}\right)\left(\frac{d w}{w}\right)}=\operatorname{res}_{d \lambda=0} \frac{d \omega_{i} d \omega_{j} d \omega_{k}}{\left(\frac{d \lambda}{\lambda}\right)\left(\frac{d P}{Y}\right)}=\sum_{\alpha} \lambda_{\alpha} \frac{\widehat{\omega}_{i}\left(\lambda_{\alpha}\right) \widehat{\omega}_{j}\left(\lambda_{\alpha}\right) \widehat{\omega}_{k}\left(\lambda_{\alpha}\right)}{P^{\prime}\left(\lambda_{\alpha}\right) / \widehat{Y}\left(\lambda_{\alpha}\right)} .
$$

Другие примеры. Основной пример, когда уравнения (129) не выполняются [18], это эллиптическая модель Калоджеро-Мозера, для которой кривая $\Sigma$ является накрытием эллиптической кривой (т.е. не гиперэллиптическая). Для таких теорий пока не построено уравнений ассоциативности, возможно, их следует связывать с эллиптическими обобщениями классов Громова-Виттена. Одна из проблем, сразу возникающих при исследовании “эллиптического” случая, - это вырожденность метрики $G$ в полном пространстве модулей (связанная с конформной инвариантностью четырехмерной модели).

В качестве других примеров решений уравнений ВДВВ (часто формальных решений, т.к. далеко не всегда известна их физическая интерпретация) можно рассматривать кривые, отвечаюшие менее известным иерархиям интегрируемых уравнений, особенностям в отмеченных точках и т.п. Например, недавно был построен пример решения [58], для которого производяший дифференциал $d S$ непосредственно выражается через функцию Бейкера-Ахиезера $d S=\Lambda d \log \Psi$. Интересно возможное обобшение решений уравнений ВДВВ на эллиптический или шестимерный случай в том смысле, в котором пятимерная теория представляет собой “цилиндрическое" $\lambda \rightarrow \log \lambda$ обобщение четырехмерной. Соответствуюшее шестимерное обобшение наивно возникает, когда параметр $\lambda$ следует считать координатой на эллиптической кривой. Сохраняются ли в этом случае уравнения ассоциативности, пока не известно. 


\section{4. ЗАКЛЮЧЕНИЕ}

В этой статье была сделана попытка представить основные положения теории интегрируемых систем, которые недавно оказались чрезвычайно полезными для понимания непертурбативных результатов в квантовой теории струн и суперсимметричной калибровочной теории. Наиболее необычное (и новое!) явление заключается в том, что классические и конечномерные интегрируемые системы эффективно описывают квантовую (бесконечномерную!) теорию поля, в которой, в частности, имеются распространяюшиеся (безмассовые) частицы и которая не является квантовой интегрируемой системой в обычном смысле этих слов.

Гипотетически возможно обобщение данного подхода на реалистические струнные модели, в первую очередь, на исследование свойств препотенциалов, возникающих в моделях струн, компактифицированных на многообразия типа многообразия Калаби-Яо. В принципе для таких моделей возможно повторить по крайней мере некоторые из сделанных выше шагов, что должно вплотную подвести к интегрируемым моделям, основанным на многомерных комплексных многообразиях (вместо $1_{\mathbf{C}}$-мерных кривых $\Sigma$ ). Такие интегрируемые системы пока практически не исследованы (тем не менее, см. $[41,59])$ и скорее всего окажутся гораздо более сложными, чем хорошо известные иерархии типа иерархий КП или Тоды.

Другое важное направление (почти не затронутое в тексте) связано с изучением эффективных теорий в пространстве-времени с компактными измерениями [60, 19]. Добавление одного компактного измерения приводит к хорошо известной "релятивизации" интегрируемых моделей [57], а также позволяет придать физический смысл дивизору на комплексной кривой соответствуюшего конечнозонного решения в терминах нелокальных наблюдаемых (петель). Таким образом, с одной стороны, это может прояснить природу возникающих интегрируемых систем, а с другой стороны, представляет собой шаг на пути к изучению препотенциалов струнных моделей (см., например, [61]), в которых появляются аналогичные (хотя и более сложные) структуры.

Несмотря на все сушествующие проблемы хочется верить, что для всех теорий, где имеется возможность получить точные непертурбативные результаты, можно найти нечто большее, чем суммирование ряда теории возмушений. Основная идея, которую мы пытались отстоять выше и которая уже подтверждена рядом примеров, заключается в правдоподобности гипотезы о том, что самосогласованная теория сама регулирует себя на малых и больших расстояниях. Похоже, что адекватньй непертурбативньй язык для эффективной формулировки самосогласованных в этом смысле теорий следует искать среди интегрируемых систем.

Автор благодарен Б. Воронову, А. Горскому, Б. Дубровину, А. Забродину, А. Лосеву, Н. Некрасову, А. Орлову, А. Рослому, И. Тютину, В. Файнбергу, В. Фоку и особенно И. Кричеверу, А. Левину, А. Миронову и А. Морозову за полезные обсуждения. Работа была частично поддержана грантом РФФИ 96-02-19085, а также грантом INTAS 93-0633.

\section{Список литературы}

[1] A. Gorsky, I. Krichever, A. Marshakov, A. Mironov, A. Morozov. Phys. Lett. B. 1995. V. 355. P. 466; hep-th/9505035.

[2] E. Martinec, N. Warner. hep-th/9509161. 
[3] T. Nakatsu, K.Takasaki. hep-th/9509162.

[4] R. Donagi, E. Witten. hep-th/9510101.

[5] E. Martinec. hep-th/9510204.

[6] A. Gorsky, A. Marshakov. hep-th/9510224; Phys.Lett. B. 1996. V. 375. P. 127.

[7] E. Martinec, N. Warner. hep-th/9511052.

[8] H. Itoyama, A. Morozov. hep-th/9511126; hep-th/9512161; hep-th/9601168.

[9] A. Marshakov. Mod. Phys. Lett. A. 1996. V. 11. P. 1169; hep-th/9602005.

[10] C. Ann, S. Nam. hep-th/9603028.

[11] A. Gorsky, A. Marshakov, A. Mironov, A. Morozov. hep-th/9603140; Phys. Lett. B. 1996. V. 380. P. 75 .

[12] A. Gorsky, A. Marshakov, A. Mironov, A. Morozov. hep-th/9604078; In: Problems in Modern Theoretical Physics. Dubna, 1996. P. 44-62.

[13] I. Krichever, D. Phong. hep-th/9604199.

[14] A. Marshakov. Preprint FIAN/TD-11/96; ITEP/TH-23/96; hep-th/9607159.

[15] A. Marshakov. Preprint FIAN/TD-16/96; ITEP/TH-47/96; hep-th/9610242.

[16] A. Marshakov, A. Mironov, A. Morozov. Preprint FIAN/TD-10/96; ITEP/TH-22/96; hep-th/9607109; Phys. Lett. B. (in print).

[17] A. Marshakov, A. Mironov, A. Morozov. Preprint FIAN/TD-15/96; ITEP/TH-46/96; hep-th/9701123.

[18] A. Marshakov, A. Mironov, A. Morozov. Preprint FIAN/TD-01/97; ITEP/TH-02/97; hep-th/9701014.

[19] N. Nekrasov. hep-th/9609219.

[20] N. Seiberg, E. Witten. Nucl.Phys. B. 1994. V. 426. P. 19; hep-th/9407087.

[21] N. Seiberg, E. Witten. Nucl. Phys. B. 1994. V. 431. P. 484; hep-th/9408099.

[22] A. Klemm, W. Lerche, S. Theisen, S. Yankielowicz. Phys. Lett. B. 1995. V. 344. P. 169; hep-th/9411048; P. Argyres, A. Faraggi. Phys. Rev. Lett. 1995. V. 73. P. 3931; hep-th/9411057.

[23] A. Hanany, Y. Oz. hep-th/9505075; P. Argyres, D. Plesser, A. Shapere. hep-th $/ 9505100$ J. Minahan, D. Nemeschansky. hep-th/9507032; P. Argyres, A. Shapere. hep-th/9509175; A. Hanany. hep-th/9509176.

[24] К. Вильсон, Джс. Когут. Ренормализационная группа и $\varepsilon$-разложение. М.: Мир, 1975.

[25] K. Ueno, K. Takasaki. Adv. Studies in Pure Math. 1984. V. 4. P. 1.

[26] M. Sato. RIMS. 1981. V. 439. P. 30; M. Sato, Y. Sato. Lect. Not. Num. Appl. Anal. 1982. V. 5. P. 259; In: Non-linear partial differential equations in applied science. Amsterdam, New York: North Holland, 1983. P. 259.

[27] G. Segal, G. Wilson. Publ. I.H.E.S. 1985. V. 61. P. 1.

[28] И. М. Кричевер. Функц. анализ и его прилож. 1977. Т. 11. С. 15; УМН. 1977. Т. 32. С. 180.

[29] В.Е. Захаров, С. В. Манаков, С. П. Новиков, Л. П. Питаевский. Теория солитонов. М.: Наука, 1980.

[30] Б. А. Дубровин. УМН. 1981. Т. 36. № 2. С. 11.

[31] Б. А. Дубровин, И. М. Кричевер, С. П. Новиков. Интегрируемые системы. І. Соврем. пробл. математики. Динамические системы, 4. М.: ВИНИТИ, 1985. С. 179.

[32] И. М. Кричевер. УМН. 1981. Т. 36. № 2. С. 12.

[33] Л. Д. Фаддеев, Л. А. Тахтаджсян. Гамильтонов подход в теории солитонов. М.: Наука, 1986.

[34] E. Sklyanin. J. Sov. Math. 1989. V. 47. Р. 2473; E. К. Склянин. Функц. анализ и его прилож. 1982. Т. 16. С. 27 ; 1983. Т. 17. С. 34.

[35] И. М. Кричевер. Функц. анализ и его прилож. 1980. Т. 14. С. 282.

[36] E. К. Склянин. Алгебра и анализ. 1994. Т. 6. С. 227; H. Braden, T. Suzuki. Lett. Math. Phys. 1994. V. 30. P. 147; B. Enriquez, V. Rubtsov. alg-geom/9503010; N. Nekrasov. hep-th/9503157; G. Arutyunov, P. Medvedev. hep-th/9511070.

[37] V. Inozemtsev. Commun. Math. Phys. 1989. V. 121. P. 629.

[38] S. Kharchev, A. Marshakov. In: String Theory, Quantum Gravity, and the Unification of Fundamental Interactions. Singapore: World Scientific, 1993. P. 331.

[39] S. Kharchev, A. Marshakov. Int. J. Mod. Phys. A. 1995. V. 10. P. 1219.

[40] И. М. Кричевер. УМН. 1989. Т. 44. № 2. С. 121. 
[41] N. Hitchin. Duke. Math. J. 1987. V. 54. P. 91.

[42] J. Fay. Theta-functions on Riemann surfaces. Lect. Notes Math. V. 352. N. Y.: Springer, 1973.

[43] Д. Мамфорд. Лекции о тэта-функциях. М.: Мир, 1988.

[44] A. Beilinson, Yu. Manin. Commun. Math. Phys. 1986. V. 107. P. 359.

[45] I. Krichever. Commun. Pure Appl. Math. 1994. V. 47. P. 437; Preprint LPTENS-92-18.

[46] И. М. Кричевер. Функц. анализ и его прилож. 1988. Т. 22. С. 37; I. Krichever. Commun. Math. Phys. 1991. V. 143. P. 415.

[47] Б. А. Дубровин, С. П. Новиков. УМН. 1989. Т. 44. № 6. С. 29.

[48] А.И. Гуревич, Л. П. Питаевский. ЖЭТФ. 1973. Т. 65. С. 590; В. Е. Захаров, С. В. Манаков, С. П. Новиков, Л. П. Питаевский. Теория солитонов. М.: Наука, 1980.

[49] B. Dubrovin. hep-th/9407018; Nucl. Phys. B. 1992. V. 379. P. 627.

[50] Yu. Manin. Frobenius manifolds, quantum cohomology, and moduli spaces. Preprint MPI, 1996.

[51] E. Witten. Surv. Diff. Geom. 1991. V. 1. P. 243; R. Dijkgraaf, E. Verlinde, H. Verlinde. Nucl. Phys. B. 1991. V. 352. P. 59.

[52] M. Kontsevich, Yu. Manin. Commun. Math. Phys. 1994. V. 164. P. 525.

[53] A. Лосев. ТМФ. 1993. T. 95. С. 307; A. Losev, I. Polyubin. Int. J. Mod. Phys. A. 1995. V. 10. P. 4161.

[54] W. Lerche, C. Vafa, N. Warner. Nucl. Phys. B. 1989. V. 324. P. 427.

[55] П. Гриффитс, Джс. Харрис. Принципы алгебраической геометрии. М.: Мир, 1982.

[56] N. Dorey, V. Khoze, M. Mattis. hep-th/9607202; hep-th/9611016; E. D'Hoker, I. Krichever, D. Phong. hep-th/9609041.

[57] S. Ruijsenaars. Finite-dimensional Soliton Systems. In: Integrable and Super-Integrable Systems. Singapore: World Scientific, 1989.

[58] I. Krichever. hep-th/9611158.

[59] E. Markman. Comp. Math. 1994. V. 93. P. 255; R. Donagi, E. Markman. Cubics, integrable systems, Calabi-Yau Threefolds, preprint; Spectral covers, algebraically completely integrable Hamiltonian systems, moduli of bundles, preprint.

[60] N. Seiberg, E. Witten. hep-th/9609219.

[61] J. Harvey, G. Moore. hep-th/9510182.

Поступила в редакцию 10. II.1997 г.

\section{A. V. Marshakov \\ ON INTEGRABLE SYSTEMS AND SUPERSYMMETRIC GAUGE THEORIES}

The properties of the $\mathcal{N}=2$ SUSY gauge theories underlying the Seiberg-Witten hypothesis are discussed. The main ingredients of the formulation of the finite-gap solutions to integrable equations in terms of complex curves and generating 1-differential are presented, the invariant sense of these definitions is illustrated. Recently found exact nonperturbative solutions to $\mathcal{N}=2$ SUSY gauge theories are formulated using the methods of the theory of integrable systems and where it is possible the parallels between standard quantum field theory results and solutions to integrable systems are discussed. 